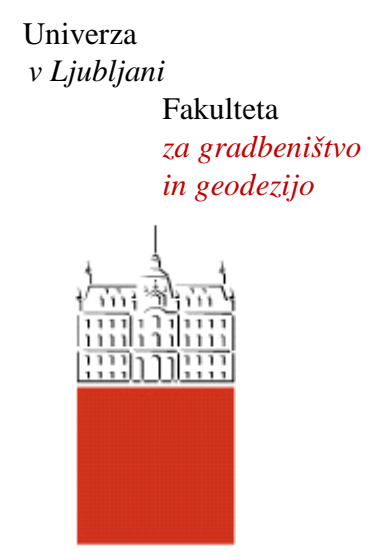

Jamova 2

1000 Ljubljana, Slovenija http://www3.fgg.uni-lj.si//

DRUGG - Digitalni repozitorij UL FGG http://drugg.fgg.uni-lj.si/

Ta članek je avtorjeva zadnja recenzirana različica, kot je bila sprejeta po opravljeni recenziji.

Prosimo, da se pri navajanju sklicujte na bibliografske podatke, kot je navedeno:
University
of Ljubljana

Faculty of

Civil and Geodetic

Engineering

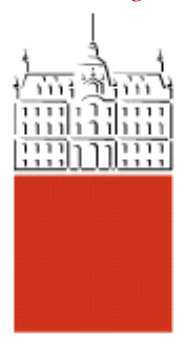

Jamova 2

SI - 1000 Ljubljana, Slovenia

http://www3.fgg.uni-lj.si/

DRUGG - The Digital Repository http://drugg.fgg.uni-lj.si/

This version of the article is author's manuscript as accepted for publishing after the review process.

When citing, please refer to the publisher's bibliographic information as follows:

Brank, B., Perić, D., Damjanić, F. 1995. On implementation of a nonlinear four node shell finite element for thin multilayered elastic shells. Comput. mech., 16, 5: 341-359.

Doi: $10.1007 / \mathrm{BF} 00350723$ 


\title{
On implementation of a nonlinear four node shell finite element for thin multilayered elastic shells
}

\author{
B. Brank ${ }^{1}$, D. Perić ${ }^{2}$ F. B. Damjanić ${ }^{1}$ \\ ${ }^{1}$ Institute of Structural and Earthquake Engineering, University of Ljubljana, Jamova 2, 61000 Ljubljana, Slovenia \\ ${ }^{2}$ Department of Civil Engineering, University of Wales, Swansea, Swansea SA2 SPP, Wales, U.K.
}

\begin{abstract}
A simple non-linear stress resultant four node shell finite element is presented. The underlying shell theory is developed from the three dimensional continuum theory via standard assumptions on the displacement field. A model for thin shells is obtained by approximating terms describing the shell geometry. In this work the rotation of the shell director is parameterized by the two Euler angles, although other approaches can be easily accommodated. A procedure is provided to extend the presented approach by including the through-thickness variable material properties. These may include a general non-linear elastic material with varied degree of orthotropy, which is typical for fibre reinforced composites. Thus a simple and efficient model suitable for analysis of multilayered composite shells is attained. Shell kinematics is consistently linearized, leading to the Newton-Raphson numerical procedure, which preserves quadratic rate of asymptotic convergence. A range of linear and non-linear tests is provided and compared with available solutions to illustrate the approach.
\end{abstract}

\section{Introduction}

Lower order elements present the majority of finite elements that are currently employed in engineering practice. The four node shell elements based on isoparametric interpolation scheme have been commonly used for the finite element implementation of different shell theories of the MindlinReissner type. In the recent works of Parisch (1991), Sansour and Bufier (1992), Sima et al. (1989a, b, 1990) and Wriggers and Gruttmann (1993) the four node elements take part in non-linear formulations capable to describe large displacements and large rotations of shells.

These finite element formulations arise from the shell theories based on the direct approach (Sima and Fox 1989a) or, alternatively, they may be derived from the three dimensional continuum theory by employing either Green-Lagrange (Parisch 1991; Stander et al. 1989) or Biot (Sansour and Bufier 1992; Wriggers and Gruttmann 1993) strain measures. The strain measures are typically restricted to be small.
A strong analogy of the formulations based on the degenerated continuum approach (e.g. Stander et al. 1989) and those based on shell theories can be noticed (see Buchter and Ramm 1992), if a certain versions of an explicit integration or a numerical integration through the shell thickness are performed in the former. For instance, Buchter and Ramm (1992) showed, that the numerical integration across the thickness in the degenerated continuum model leads to the strain tensor, which is consistent with the so-called first approximation of a geometrically non-linear shell theory for small strains including transverse shear deformations (Ba9ar and Ding 1990, Naghdi 1972).

The displacement based formulations suffer from the so-called transverse shear locking, a phenomena which is closely connected with the underlying assumptions typical for the shell theories of the Mindlin-Reissner type. It is therefore essential that the solutions are based on mixed variational formulations. A current 'standard' to avoid transverse shear locking is the so-called ANS (Assumed Natural Strain) approach, first suggested by Dvorkin and Bathe (1984). Other interesting schemes, arising from mixed variational formulations, that improve the membrane and/or bending behaviour of the four node element (especially for a coarse meshes), can be found in the works of Simo et al. (1989b, 1990), Pian and Sumihara (1984) and recently Andelfinger and Ramm (1993). Andelfinger and Ramm (1993) use the so-called EANS (Enhanced Assumed Natural Strain) approach. Another type of possible errors arise from the type of discretisation. In that sense an attempt to modify the four node elements to be capable to describe curved geometries was made by Gebhardt and Schweizerhof (1993).

In many non-linear shell theories the description of the rigid motion of the shell normal is required. Large rotations therefore take an important part in the computational models. Moreover, they are treated as a critical part of the finite element implementation of the variational formulation. Large rotation formulations are mostly based either on an elementary rotations (Stander et al. 1989, Wriggers and Gruttmann 1993) or on a rotational vector (Parisch 1991, Sansour and Bufier 1992, Simo and Fox 1989a). The elementary rotation is considered to be a rotation around a fixed axis. A sequence of elementary rotations then may be used to describe rotation in a three- 
dimensional space. Unfortunately this approach suffers from singularities which depend on numerical implementation. In contrast, the rotational vector is an eigenvector of a rotation matrix and together with a rotation angle, it may describe any rotation without singularities.

Our aim in this paper is to present a geometrically non-linear formulation for a simple four-node shell element, arising from the shell theory (initial version is presented by Brank et al.

1993). The Green-Lagrange strain measures are used for that purpose coupled with suitable assumptions on the shell geometry. A formulation based on elementary rotations, i.e. rotations around fixed axes, is used, which, although less general, suffices for the present purpose. The formulation is based on two independent angles, first presented by Ramm in 1976 (see e.g. Büchter and Ramm (1992), Ramm and Matzenmiller (1986), Stander et al. (1989)). This parametrisation of large rotations enables five nodal degrees of freedom and restricts the accompanied finite element to smooth shells analysis. The transverse shear locking is avoided by 'standard' ANS approach (Bathe and Dvorkin 1985, Dvorkin and Bathe 1984). A variational background of the ANS approach is given by Simo and Hughes (1986). An important computational aspect of the isoparametric shell elements is also a treatment of membrane locking - a phenomena which is associated with a parasitic membrane strains under pure bending conditions. Since elements with bi-linear interpolation do not show a particular sensitivity to this defect, a displacement formulation is used for the membrane and bending parts of a variational formulation. Local Cartesian frames are defined at numerical integration points in order to simplify the expressions.

A procedure is provided to extend the presented approach by including the through-thickness variable material properties. These may include a general non-linear elastic material with varied degree of orthotropy, which is typical for fibre reinforced composites. Thus a simple and efficient model suitable for analysis of multilayered composite shells is attained.

From the computational point of view, a robust and efficient algorithm with high rate of convergence is required. Consistent linearisation of the shell kinematics leading to the NewtonRaphson numerical procedure is therefore performed.

\section{2}

\section{Shell kinematics and strain measures}

Following a traditional approach outlined by Green and Zerna (1968), from the outset, the shell $\mathscr{S}$ is treated as a body embedded in the three-dimensional Euclidean space $\mathbb{R}^{3}$. Shell deformation is assumed to obey certain kinematic assumptions described in detail below. Furthermore, the shell reference (middle) surface is assumed to be smooth, continuous and differentiable. The shell thickness is assumed to be constant or only slightly varying.

\section{1}

\section{Preliminaries}

Attention is restricted to the material description of the Lagrangian type. Material points of the shell body are identified by curvilinear (convected) coordinates $\xi:=\left\{\xi^{1}, \xi^{2}, \xi\right\}$, attached to the body. Two coordinates, $\xi^{1}$ and $\xi^{2}$, parametrize the mid-surface of the shell and the third one, $\xi$, is a parameter which defines the shell thickness. The position vectors in the reference and deformed configuration, along with the displacement vector, are related to the fixed Euclidean frame with base vectors $E_{l}$. This is a practice which makes shell theories well suited for the numerical treatment, since quantities such as Cristoffel's symbols need not be computed.

Two basic assumptions (classical for the shell theories that do not define a thickness stretch as an independent kinematic variable) define the displacement field: (i) a linear variation of the displacements across the shell thickness and (ii) an inextensibility of a shell director field. In this way the shell director becomes a unit vector normal to the shell mid-surface at the reference configuration, but not necessarily normal to the deformed mid-surface. This enables the presence of transverse shear strains and allows the use of $C^{\circ}$ shape functions within the finite element computations.

Any configuration of the shell body in the Euclidian space can be completely described by the position of the mid-surface and the position of the shell director. Moreover, the kinematics of the shell director can be viewed as a rigid motion of a unit vector. A material point of the shell body at the deformed and reference configurations is defined by the mid-surface point and a distance along the shell director, with position vectors as follows

$r\left(\xi^{\alpha}, \xi\right)=x\left(\xi^{\alpha}\right)+\xi t\left(\xi^{\alpha}\right), \quad R\left(\xi^{\alpha}, \xi\right)=X\left(\xi^{x}\right)+\xi T\left(\xi^{\alpha}\right)$,

where

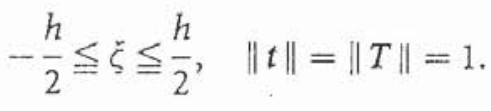

Here, $h$ is the shell thickness, $X$ and $x$ denote the mid-surface position vector at the reference and current configuration, respectively, while $T$ and $t$ denote the shell director at initial and deformed configuration, respectively. Greek indices are either 1 or 2 and Latin indices run from 1 to 3 . Cartesian structure of the ambient space allows for the following decomposition

$x\left(\xi^{\alpha}\right)=X\left(\xi^{x}\right)+u\left(\xi^{\alpha}\right)$,

where $u$ is the displacement vector of the mid-surface. From $(1-3)$ the displacement field $w\left(\xi^{x}, \xi\right):=r\left(\xi^{\alpha}, \xi\right)-R\left(\xi^{\alpha}, \xi\right)$ can be expressed as

$w\left(\xi^{x}, \xi\right)=u\left(\xi^{x}\right)+\xi\left(t\left(\xi^{x}\right)-T\left(\xi^{x}\right)\right)$,

which indicates a linear variation of the displacement field across the shell thickness. Clearly, the mid-surface displacement $u$ and the director displacement $\left(t\left(\xi^{x}\right)-T\left(\xi^{x}\right)\right)$ are independent. This fact allows for a simple description of transverse shear strains and simplifies the finite element interpolation.

\section{2}

\section{Metric tensors}

Following standard approach the covariant (convected) basis at any material point is obtained as

$g_{x}=\frac{\partial r}{\partial \xi^{x}}, \quad g_{3}=\frac{\partial r}{\partial \xi^{\prime}}, \quad$ and $\quad G_{x}=\frac{\partial R}{\partial \xi^{x}}, \quad G_{3}=\frac{\partial R}{\partial \xi}$. 
and reference configuration can then be expressed as

$$
\begin{aligned}
& t=Q(\psi, \omega) E_{2}=Q_{1}(\psi) Q_{3}\left(\omega-\frac{\pi}{2}\right) E_{2}, \\
& T=Q(\Psi, \Omega) E_{2}=Q_{1}(\Psi) Q_{3}\left(\Omega-\frac{\pi}{2}\right) E_{2} .
\end{aligned}
$$

The orthogonal matrices $Q(\psi, \omega)$ and $Q(\Psi, \Omega)$ rotate $E_{2}$ to $t$ and $T$, respectively. Here $Q_{1}(\psi)\left(Q_{1}(\Psi)\right)$ and $Q_{3}(\omega)\left(Q_{3}(\Omega)\right)$ define $(3 \times 3)$ orthogonal matrices. For the current placement of the shell, for instance, they rotate Euclidean basis, along with vectors defined in that basis, around the fixed axes, defined by the base vectors $E_{1}$ and $E_{3}$, for the angles $\psi$ and $\omega$

$$
Q_{1}(\psi)=\left[\begin{array}{ccc}
1 & 0 & 0 \\
0 & \cos (\psi) & -\sin (\psi) \\
0 & \sin (\psi) & \cos (\psi)
\end{array}\right]
$$

$Q_{3}(\omega)=\left[\begin{array}{ccc}\cos (\omega) & -\sin (\omega) & 0 \\ \sin (\omega) & \cos (\omega) & 0 \\ 0 & 0 & 1\end{array}\right]$

Description of the motion is as follows: rotation of $E_{2}$ around $E_{1}$ is followed by the rotation around the new position of $E_{3}$. This procedure is equivalent to the sequence of rotations around fixed axes as given in (13). This description and Fig. 1 help to understand that angles at the deformed configuration can be evaluated by a simple summation of initial angles, $\Psi, \Omega$, and the shell director 'rotations', $\alpha, \beta$, around axes $f_{\alpha}$ and $f_{\beta}$

$f_{\alpha}=E_{1}, \quad f_{\beta}=E_{1} \times t$.

In Fig. 1 it can be seen, that $\Psi$ and $\alpha$ are coplanar, as well as $\Omega$ and $\beta$, so that the following relationships are valid

$\psi=\Psi+\alpha, \quad \omega=\Omega+\beta$.

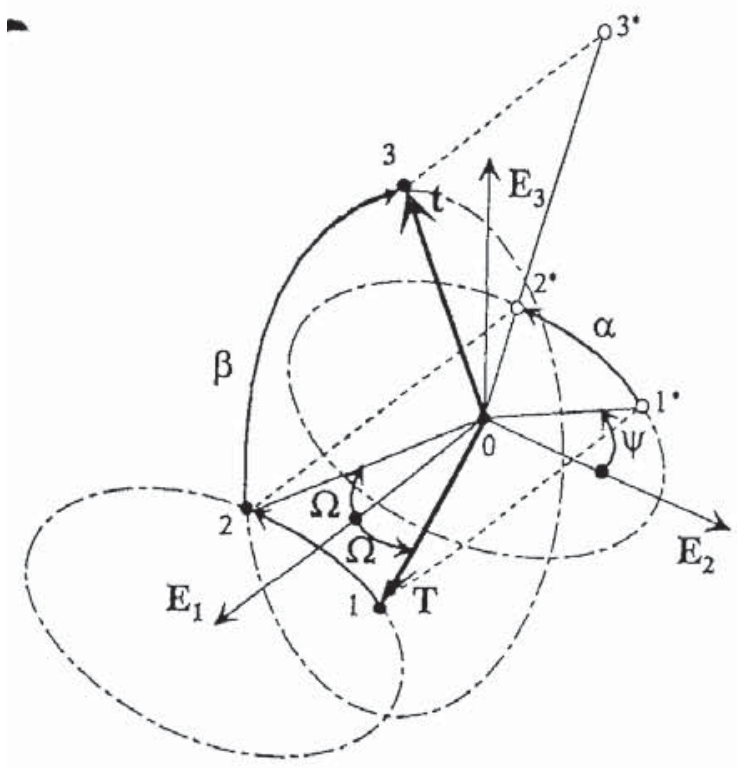

Fig. 1. Shell director at initial and deformed configuration
Finally, for chosen parametrisation, the explicit form of $t$ reads as

$t=\left\{\begin{array}{c}\cos (\omega) \\ \cos (\psi) \sin (\omega) \\ \sin (\psi) \sin (\omega)\end{array}\right\}$

Note, that the axis of rotation, $f_{\beta}$, cannot be defined when $\omega$ approaches to $\omega=(k-1) \pi, k \in \mathbb{Z}$.

\section{3}

\section{The principle of virtual work}

Having defined strain measures, we proceed with the expression for the principle of virtual work. It is set in the reference configuration in accordance with the chosen stress and strain measures.

\section{1}

\section{The principle of virtual work in stress resultants}

A stress tensor energy conjug ite to the Green-Lagrange strain tensor is the second Piola-Kirchhoff stress tensor. In the convected basis it may be expressed as $S=S^{i j} G_{i} \otimes G_{i}$. The three dimensional expression for the principle of virtual work then reads as

$$
\begin{aligned}
G(r, \delta r)= & \int_{S_{o}}\left(\int_{-h / 2}^{+h / 2}\left(S^{\alpha \beta}\left(\delta \varepsilon_{\alpha \beta}+\xi \delta \kappa_{\alpha \beta}\right)+S^{\alpha 3} \delta \gamma_{\alpha}\right) \mu \mathrm{d} \xi\right) \mathrm{d} S_{o} \\
& -G_{\text {ext }}(\delta r)=0,
\end{aligned}
$$

where $S_{o}$ denotes the shell mid-surface area, $G_{\text {ext }}$ being the work of external forces and the volume element is $\mathrm{d} V_{o}=\mu d \xi \mathrm{d} S_{o}$

(Naghdi 1972) with $\mu=\sqrt{G / A}$, where $G=\operatorname{det}\left[G_{i j}\right]$ and $A=\operatorname{det}\left[A_{\alpha \beta}\right]$. Integration across the shell thickness gives

$$
G(r, \delta r)=\int_{S^{o}}\left(n^{\alpha \beta} \delta \varepsilon_{\alpha \beta}+m^{\alpha \beta} \delta \kappa_{\alpha \beta}+q^{\alpha} \delta \gamma_{\alpha}\right) \mathrm{d} S_{o}-G_{\text {ext }}(\delta r)=0,
$$

where $n^{\alpha \beta}, m^{\alpha \beta}$ and $q^{\alpha}$ represent the components of the so-called 'effective part' of the stress resultants. They are symmetric, (opposite to their physical counterparts, cf. Simo and Fox 1989a), and expressed as

$$
n^{\alpha \beta}=\int_{-h / 2}^{+h / 2} S^{\alpha \beta} \mu \mathrm{d} \xi, \quad m^{\alpha \beta}=\int_{-h / 2}^{+h / 2} S^{\alpha \beta} \mu \xi \mathrm{d} \xi, \quad q^{\alpha}=\int_{-h / 2}^{+h / 2} S^{\alpha 3} \mu \mathrm{d} \xi .
$$

Equation (19) is a non-linear function in kinematic variables. In computations an iterative solution procedure is performed, which usually employs a linearisation of (19). In this paper, the linearisation is performed after the finite element discretisation.

\section{2}

\section{Variation of the strain measures}

Strain measure variations are obtained by taking the directional derivative in the direction of the virtual displacements, $\delta u$, and virtual 'rotations', $\delta t$, given by $(\delta u, \delta t):=\left.(\mathrm{d} / \mathrm{d} \varepsilon)\right|_{\varepsilon=0}\left(x_{\varepsilon}, t_{\varepsilon}\right)$. This 
results in

$$
\begin{aligned}
\delta \varepsilon_{\alpha \beta} & :=\mathrm{D}\left(\varepsilon_{\alpha \beta}\right)[\delta u, \delta t]=\frac{1}{2}\left(x_{, \alpha} \cdot \delta u_{, \beta}+x_{, \beta} \cdot \delta u_{, \alpha}\right), \\
\delta \gamma_{\alpha} & :=\mathrm{D}\left(\gamma_{\alpha}\right)[\delta u, \delta t]=\left(x_{, \alpha} \cdot \delta t+\delta u_{, \alpha} \cdot t\right) \\
\delta \kappa_{\alpha \beta} & :=\mathrm{D}\left(\kappa_{\alpha \beta}\right)[\delta u, \delta t] \\
& =\frac{1}{2}\left(x_{, \alpha} \cdot \delta t_{, \beta}+x_{, \beta} \cdot \delta t_{, \alpha}+\delta u_{, \alpha} \cdot t_{, \beta}+\delta u_{, \beta} \cdot t_{, \alpha}\right)
\end{aligned}
$$

Details of this procedure are provided below in Section 6 .

\section{4}

\section{Constitutive equations}

At the outset existence of a hyperelastic stored energy function is assumed. The stress components can then be obtained as derivatives of the strain energy function by the corresponding strain components. Here a simple hyperelastic isotropic constitutive law will be employed given by the St.

Venant-Kirchhoff strain energy function. For the strain -measures defined in (12), the thickness stretch $\varepsilon_{33}$ does not appear as a kinematic variable. Hence, the stretch in $\xi$ direction is obtained in a standard way by employing an assumption of sro stress in that direction. This is simply performed by the condensation of a material law. Written in the curvilinear coordinates, the constitutive relations for the thin shells take the standard form, which is obtained from an integration of stresses across the thickness, (20). If the variation of the metric across the thickness is ignored, i.e. $G^{\alpha \beta} \approx A^{\alpha \beta}$ is assumed (in that case $\mu$ equals to 1 ), the following relations are obtained

$$
S^{\alpha \beta}=\frac{E}{1-v^{2}} H^{\alpha \beta \gamma \delta}\left(\varepsilon_{\gamma \delta}+\xi \kappa_{\gamma \delta}\right), \quad S^{x 3}=\kappa G A^{\alpha \beta} \gamma_{\beta},
$$

which result in

$$
\begin{aligned}
& n^{\alpha \beta}=\frac{E h}{1-v^{2}} H^{\alpha \beta \gamma \delta} \varepsilon_{\gamma \delta}, \quad m^{\alpha \beta}=\frac{E h^{3}}{12\left(1-v^{2}\right)} H^{\alpha \beta \gamma \delta} \kappa_{\gamma \delta}, \\
& q^{\alpha}=\kappa G h A^{\alpha \beta} \gamma_{\beta},
\end{aligned}
$$

\section{where}

$$
H^{\alpha \beta \gamma \delta}=v A^{\alpha \beta} A^{\gamma \delta}+\frac{1}{2}(1-v)\left(A^{\alpha \gamma} A^{\beta \delta}+A^{\alpha \delta} A^{\beta \gamma}\right) .
$$

Here, $E, G, v$ and $\kappa$ are the elastic (Young's) modulus, shear modulus, Poisson's ratio and shear correction factor, respectively. Note, that in (23) the contravariant mid-surface metric, $A^{\alpha \beta}$, is used instead of $G^{x \beta}$, since the assumption of constant through-thickness metric was adopted. The consequence is that membrane-bending coupling vanishes. In this case membrane, bending and transverse shear stress resultants are related only to their conjugate strain measures $\varepsilon_{\alpha \beta}, \kappa_{\alpha \beta}, \gamma_{\beta}$, which leads to the block-diagonal constitutive matrix (see Appendix).

Alternatively, if an integration across the thickness in (20) is carried out numerically, then the variation of metric with respect to $\xi,(8)$, can be easily taken into account. When material characteristics vary across the thickness, as in the case of materially non-linear behaviour, then numerical integration across the thickness presents a natural choice. As a result coupled membrane-bending action is achieved.

In Appendix B, details are provided for a procedure that extends the above described approach to the multilayered elastic composite shells by including the through-thickness variable material properties. The resulting model then becomes suitable for analysis of laminated shell structures that may be composed of perfectly bonded layers of elastic materials with variable degree of orthotropy, typically encountered with fibre reinforced composites.

\section{5}

\section{Finite element discretization}

The displacement vector and rotational parameters are approximated using the isoparametric approach.

\section{1}

\section{Mid-surface interpolation}

The geometry of the shell and the kinematic variables are interpolated with standard bilinear functions $N^{I}\left(\xi^{1}, \xi^{2}\right)=$ $\frac{1}{4}\left(1+\xi^{1} \xi_{I}^{1}\right)\left(1+\xi^{2} \xi_{I}^{2}\right)$, where $(I=1,2,3,4),\left(\xi_{f}^{1}=-1,+1,+1\right.$, $-1)$ and $\left(\xi_{I}^{2}=-1,-1,+1,+1\right)$. When convenient the coordinates, $\xi$ and $\eta$, will be used to denote the curvilinear coordinates, $\xi^{1}$ and $\xi^{2}$, respectively. The mid-surface quantities are interpolated as

$$
\begin{aligned}
& X=\sum_{I=1}^{4} N^{I}\left(\xi^{1}, \xi^{2}\right) X_{I}, \quad T=\sum_{I=1}^{4} N^{I}\left(\xi^{1}, \xi^{2}\right) T_{I}, \\
& u=\sum_{I=1}^{4} N^{I}\left(\xi^{1}, \xi^{2}\right) u_{I}, \quad t=\sum_{I=1}^{4} N^{I}\left(\xi^{1}, \xi^{2}\right) t_{I} .
\end{aligned}
$$

In view of (4), the interpolation of shell director can be considered as an interpolation of displacement $w$. Of course, rotational variables itself can be interpolated instead of the shell director components (Büchter and Ramm 1992). However, such an approximation complicates the derivation of the tangent matrix. Although the full assessement requires further research, it appears that the interpolation of rotation variables leads to better results for coarse meshes (Büchter and Ramm 1992).

\section{2}

\section{Reference nodal director}

Description of element geometry requires the shell director position at each nodal point. Specifying its data as an input or using the normals to the exact shell geometry is impractical. In the present formulation the reference nodal director, $T_{l}$, is obtained by averaging the nodal normals of adjoining elements, as suggested Parisch (1991) and Stander et al. (1989).

The element nodal normal is given by the vector product of the corresponding element edge vectors

$T_{I}^{e}=\left(X_{J}-X_{I}\right) \times\left(X_{K}-X_{I}\right)$,

where $I, J$ and $K$ are given in the following order: $(I=1,2,3,4)$, $(J=2,3,4,1)$ and $(K=4,1,2,3)$. The averaged nodal normal is evaluated as

$\tilde{T}_{I}=\frac{1}{N_{\text {lel }}} \sum_{e=1}^{N_{l e l}} T_{l}^{e}, \quad T_{I}=\frac{\tilde{T}_{I}}{\left\|\tilde{T}_{I}\right\|}$, 
where $N_{t e l}$ is a number of adjoining elements at node $I$. The angles $\Psi^{t}$ and $\Omega^{t}$ are easily obtained with $T_{l}$ defined.

Remark 5.1 Note, that the averaging of element normals causes the loss of normality of the interpolated shell director $\left(24_{2}\right)$ to the finite element approximation of the shell mid-surface. Thus, the interpolated shell director is not normal to the mid-surface base vectors, $X_{x,}$, at the integration points. However, it seems that no simple approach to reduce this defect exists for the 5 parameter shell theory and for the four node element with 20 degrees of freedom. A procedure described by Gebhradt and Schweizerhof (1993) leads to severe membrane locking. When 6 degrees of freedom per node are defined, e.g. Parisch (1991), the element nodal normals can be used for the interpolation over the element. This gives significantly better approximation of the shell director. However it produces singular stiffness matrix for coplanar elements and requires a special care.

\section{3}

\section{Mid-surface derivatives}

The derivatives of shape functions due to $\xi^{1}$ and $\xi^{2}$ are

$$
\begin{aligned}
& X_{, x}=\sum_{l=1}^{4} N_{, \alpha}^{l} X_{l}, \quad T_{, x}=\sum_{i=1}^{4} N_{, x}^{l} T_{l}, \\
& u_{, x}=\sum_{l=1}^{4} N_{, x}^{l} u_{l}, \quad t_{, x}=\sum_{l=1}^{4} N_{, \alpha}^{l} t_{l} .
\end{aligned}
$$

We remark again, that in (24) and (27) the components of the shell director $T$, and $t_{f}$ are interpolated rather than the rotational variables $\Psi^{I}, \Omega^{I}$ and $\alpha^{l}, \beta^{l}$.

\section{4}

\section{Transverse shear interpolation}

The transverse shear strain field is given by an interpolation originally proposed by Dvorkin and Bathe (1984). Such an interpolation has become a 'standard' for the four node shell elements, in order to avoid the transverse shear locking phenomenon. Transverse strain measures are evaluated, using (12), only at the points A, B, C and D (Fig. 2). Their interpolation across the element is given by

$$
\begin{aligned}
& \gamma_{1}=\frac{1}{2}\left(1-\xi^{2}\right) \gamma_{1}^{\wedge}+\frac{1}{2}\left(1+\xi^{2}\right),{ }_{i 1}^{C},
\end{aligned}
$$

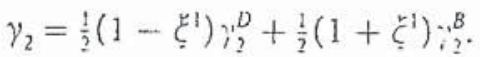

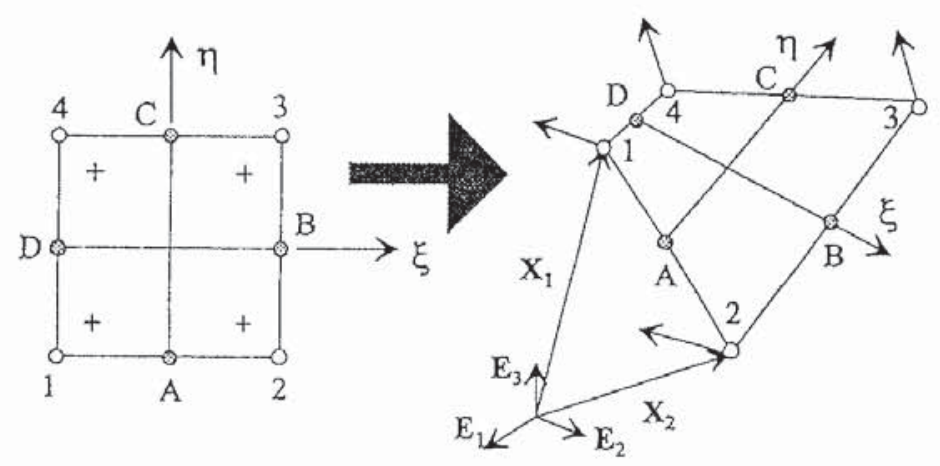

Fig. 2. Four node element
In the computational process the explicit evaluation of transverse strain at the points $A, B, C$ and $D$ is not necessary (see Appendix A).

\section{6}

\section{Linearization aspects}

Using interpolation functions, given in Sect. 5, a discrete formulation of the first variation of a mixed functional can be constructed. This equation is a non-linear function in kinematic variables (nodal displacements and 'rotations'). Therefore the linearisation of this equation is required within the iterative Newton-Raphson solution procedure. It should be observed that the linearisation is performed after the finite element discretisation.

\section{1}

\section{Linearisation of kinematic variables}

Linearisation of kinematic variables at a given configuration $r=(x, t)$ is performed by a systematic application of the directional derivative to a one-parameter family of configurations described by $r_{\varepsilon}=\left(x_{\varepsilon}, t_{\varepsilon}\right)$. The incremental quantities are then obtained by an ordinary differentiation with respect to $\varepsilon$ as

$(\Delta x, \Delta t):=\left.\frac{\mathrm{d}}{\mathrm{d} \varepsilon}\right|_{\varepsilon=0}\left(x_{\varepsilon}, t_{\varepsilon}\right)$.

Linear structure of $\mathbb{R}^{3}$ provides a simple expression for the curve $x_{t}$

$x_{\varepsilon}=x+\varepsilon \Delta u$,

while the one-parameter curve $t_{r}$ is obtained as

$t_{\varepsilon}=t(\psi+\varepsilon \Delta \alpha, \omega+\varepsilon \Delta \beta)$.

It then follows, trivially, that

$\Delta x=\left.\frac{\mathrm{d}}{\mathrm{d} \varepsilon}\right|_{\varepsilon=0} x_{\varepsilon}=\Delta u$.

By employing (31) and the expression (17) for $t$ the differentiation of the one-parameter curve $t_{\varepsilon}$ results in

$\Delta t=\left.\frac{\mathrm{d}}{\mathrm{d} \varepsilon}\right|_{\varepsilon=0} t_{\varepsilon}=\Lambda\left\{\begin{array}{l}\Delta \alpha \\ \Delta \beta\end{array}\right\}$,

where the matrix $\Lambda_{(3 \times 2)}$ is given by

$\Lambda=\left[\begin{array}{cc}0 & -\sin (\omega) \\ -\sin (\psi) \sin (\omega) & \cos (\psi) \cos (\omega) \\ \cos (\psi) \sin (\omega) & \sin (\psi) \cos (\omega)\end{array}\right]_{(3 \times 2)}$.

It can easily be checked that $\Delta t \cdot t=0$ as is required.

One should also note that the second derivative of the above expressions required within the Newton-Raphson iterative process may be obtained as

$\mathrm{D}(\delta t)[\Delta t]=\left.\frac{\mathrm{d}}{\mathrm{d} \varepsilon}\right|_{\varepsilon=0}(\delta t)_{\varepsilon}=: \Delta(\delta t)=\left.\frac{\mathrm{d}}{\mathrm{d} \varepsilon}\right|_{\varepsilon=0} \Lambda_{\varepsilon}\left\{\begin{array}{l}\delta \alpha \\ \delta \beta\end{array}\right\}$. 
Details of the above differentiation procedure are provided in the Appendix A. In contrast, $\mathrm{D}(\delta u)[\Delta u, \Delta t]$ is clearly zero.

\section{2}

\section{Newton-Raphson method}

The definition of the displacement based problem is: Find a configuration $r$, such that $G(r, \delta r)=0$ for any $\delta r$. Since the solution of the above nonlinear problem is pursued by employing the Newton-Raphson method a linearisation of $G(r, \delta r)$ is required. The Newton-Raphson procedure at the configuration $r=\hat{r}$ may be expressed in the form

$\mathrm{D} G\left(\hat{f}^{(k)}, \delta r\right)\left[\Delta r^{(k)}\right]=-G\left(\hat{f}^{(k)}, \delta r\right)$,

where the left hand side of (36) supplies the tangent stiffness operator while the right side has a standard interpretation as the unbalanced (residual) forces.

\section{3}

\section{Linearisation of the virtual work functional}

Assuming the conservative loading, the tangent stiffness operator can be obtained by the directional derivative of the 'internal virtual work' in the direction $\Delta r^{e}=\left(\Delta u^{e}, \Delta t^{e}\right)^{1}$ of incremental displacements and incremental 'rotations' at the discrete (nodal) points. The tangent stiffness operator may be conventionally split into a material and geometric part

$\mathrm{D} G(r, \delta r)[\Delta r]=\mathrm{D}_{M} G(r, \delta r)[\Delta r]+\mathrm{D}_{G} G(r, \delta r)[\Delta r]$,

where

$$
\begin{aligned}
& \mathrm{D}_{M} G(r, \delta r)[\Delta r] \\
& \quad=\int_{S_{o}}\left(\mathrm{D} n^{\alpha \beta}[\Delta r] \delta \varepsilon_{\alpha \beta}+\mathrm{D} m^{\alpha \beta}[\Delta r] \delta \kappa_{\alpha \beta}+\mathrm{D} q^{\alpha}[\Delta r] \delta \gamma_{\alpha}\right) \mathrm{d} S_{o} \\
& \mathrm{D}_{G} G(r, \delta r)[\Delta r] \\
& \quad=\int_{S_{o}}\left(n^{\alpha \beta} \mathrm{D}\left(\delta \varepsilon_{\alpha \beta}\right)[\Delta r]+m^{\alpha \beta} \mathrm{D}\left(\delta \kappa_{\alpha \beta}\right)[\Delta r]+q^{\alpha} \mathrm{D}\left(\delta \gamma_{\alpha}\right)[\Delta r]\right) \mathrm{d} S_{o} .
\end{aligned}
$$

are, respectively, the material and geometric stiffness operators, and $S_{o}$ is a mid-surface area of the finite element.

\section{4}

\section{Derivatives of strain measure variations}

The directional derivative of strain measure variations $(\delta \varepsilon, \delta \gamma, \delta \boldsymbol{\kappa})$ in the direction of $\Delta r=(\Delta u, \Delta t)$ may be expressed as

$\mathrm{D}\left(\delta \varepsilon_{\alpha \beta}\right)[\Delta u, \Delta t]=\frac{1}{2}\left(\delta u_{, \alpha} \cdot \Delta\left(u_{, \beta}\right)+\Delta\left(u_{, \alpha}\right) \cdot \delta u_{, \beta}\right)$,

$\mathrm{D}\left(\delta \gamma_{\alpha}\right)[\Delta u, \Delta t]=\left(\Delta\left(u_{, \alpha}\right) \cdot \delta t+\delta u_{, \alpha} \cdot \Delta t+x_{, \alpha} \cdot \mathrm{D}(\delta t)[\Delta t]\right)$,

$\mathrm{D}\left(\delta \kappa_{\alpha \beta}\right)[\Delta u, \Delta t]=\frac{1}{2}\left(\Delta\left(u_{, \alpha}\right) \cdot \delta t_{, \beta}+\Delta\left(u_{, \beta}\right) \cdot \delta t_{, \alpha}+\delta u_{, \alpha} \cdot \Delta\left(t_{, \beta}\right)\right.$

$$
\begin{aligned}
& +\delta u_{, \beta} \cdot \Delta\left(t_{, \alpha}\right)+x_{, \alpha} \cdot D\left(\delta t_{\beta}\right)[\Delta t] \\
& \left.+x_{, \beta} \cdot \mathrm{D}\left(\delta t_{, \alpha}\right)[\Delta t]\right) .
\end{aligned}
$$

\footnotetext{
${ }^{1}$ To simplify notation in the following the superscript $(\bullet)^{t}$ denoting
} element quantities is omitted.

\section{5}

\section{Material stiffness operator}

The material stiffness operator arises from linearisation of the constitutive equations at the fixed geometry. For hyperelastic isotropic material it is recovered from the first integral in (38) by the following relations

$$
\begin{aligned}
& \mathrm{D} n^{\alpha \beta}[\Delta u, \Delta t] \delta \varepsilon_{\alpha \beta}=\mathrm{H}_{m}^{\alpha \beta \gamma \delta} \Delta \varepsilon_{\gamma \delta} \delta \varepsilon_{\alpha \beta}, \\
& \mathrm{D} m^{\alpha \beta}[\Delta u, \Delta t] \delta \kappa_{\alpha \beta}=H_{b}^{\alpha \beta \gamma \delta} \Delta \kappa_{\gamma \delta} \delta \kappa_{\alpha \beta}, \\
& \mathrm{D} q^{\alpha}[\Delta u, \Delta t] \delta \gamma_{\alpha}=\mathrm{H}_{s}^{\alpha \beta} \Delta \gamma_{\beta} \delta \gamma_{\alpha},
\end{aligned}
$$

where $\mathrm{H}_{m}^{\alpha \beta \gamma \delta}, \mathrm{H}_{b}^{\alpha \beta \gamma \delta}$ and $\mathrm{H}_{s}^{\alpha \beta}$ are membrane, bending and transverse shear parts of symmetric constitutive tensor defined by Eqs. (22) and (23). Note that $\delta \varepsilon_{\alpha \beta}, \delta \kappa_{\alpha \beta}$ and $\delta \gamma_{\alpha}$ are given by (21). Similar expressions are valid for $\Delta \varepsilon_{\alpha \beta}, \Delta \kappa_{\alpha \beta}$ and $\Delta \gamma_{\alpha}$.

The stiffness operator for multilayered composite shells is provided in the Appendix $B$.

\section{6}

\section{Geometric stiffness operator}

The geometric stiffness operator arises from the linearisation of the geometric part, when holding the material part fixed. It is recovered from the second integral in (38) by employing the following relationships

$$
\begin{aligned}
n^{\alpha \beta} \mathrm{D}\left(\delta \varepsilon_{\alpha \beta}\right)[\Delta u, \Delta t]= & \sum_{I=1}^{4} \sum_{K=1}^{4} \delta u_{I} \cdot \Delta u_{K}\left(\frac{1}{2} n^{\alpha \beta}\left(N_{, \alpha}^{K} N_{, \beta}^{I}+N_{, \beta}^{K} N_{, \alpha}^{I}\right)\right), \\
m^{\alpha \beta} \mathrm{D}\left(\delta \kappa_{\alpha \beta}\right)[\Delta u, \Delta t]= & \sum_{I=1}^{4} \sum_{K=1}^{4} \delta t_{I} \cdot \Delta u_{K}\left(\frac{1}{2} m^{\alpha \beta}\left(N_{, \alpha}^{K} N_{, \beta}^{I}+N_{, \beta}^{K} N_{, \alpha}^{I}\right)\right) \\
& +\sum_{I=1}^{4} \sum_{K=1}^{4} \delta u_{i} \cdot \Delta t_{K}\left(\frac{1}{2} m^{\alpha \beta}\left(N_{, \alpha}^{I} N_{, \beta}^{K}+N_{, \beta}^{I} N_{, \alpha}^{K}\right)\right) \\
& +\sum_{I=1}^{4} \Delta\left(\delta t_{I}\right) \cdot\left(\frac{1}{2} m^{\alpha \beta}\left(x_{, \alpha} N_{, \beta}^{I}+x_{, \beta} N_{, \alpha}^{I}\right)\right),
\end{aligned}
$$

$$
\begin{aligned}
q^{\alpha} \mathrm{D}\left(\delta \gamma_{\alpha}\right)[\Delta u, \Delta t]= & \sum_{I=1}^{4} \sum_{K=1}^{4} \delta t_{I} \cdot \Delta u_{K}\left(q^{\alpha} N_{, \alpha}^{K} N^{I}\right) \\
& +\sum_{I=1}^{4} \sum_{K=1}^{4} \delta u_{I} \cdot \Delta t_{K}\left(q^{\alpha} N_{, \alpha}^{I} N^{K}\right) \\
& +\sum_{l=1}^{4} \Delta\left(\delta t_{I}\right) \cdot\left(q^{\alpha} x_{, \alpha} N^{I}\right) .
\end{aligned}
$$

\section{7}

Remarks on finite element implementation

In this section a Cartesian coordinate system is introduced at the mid-surface integration points, and vectors and matrices are defined that will be required in the finite element

implementation. Since all quantities are derived in curvilinear coordinates, the construction of the local Cartesian frame is not necessary. However the expressions in such a system take a simple form, and they will provide a basis for future inelastic material description within the present shell formulation. 
Let $\hat{e}_{1}$ define unit base vectors of a local Cartesian coordinate system $\hat{x}^{\prime},\left(\hat{x}^{1} \equiv \hat{x}, \hat{x}^{2} \equiv \hat{y}, \hat{x}^{3} \equiv \hat{z}\right)$, such that

\section{Numerical examples}

$\hat{e}_{1} \cdot \hat{e}_{2}=0, \quad \hat{e}_{3}=\hat{e}_{1} \times \hat{e}_{2}=\frac{X_{, 1} \times X_{, 2}}{\left|X_{, 1} \times X_{, 2}\right|}$.

Note, that $\hat{e}_{1}$ and $\hat{e}_{2}$ can be any orthogonal set of vectors, which satisfy the condition (42). The introduced change of coordinates requires transformations for partial differentiation, which take the standard form: Using the chain rule we get (in matrix form)

$\left\{\frac{\partial}{\partial \hat{x}^{\beta}}\right\}=\left[\frac{\partial \xi^{\alpha}}{\partial \hat{x}^{\beta}}\right]^{t}\left\{\frac{\partial}{\partial \xi^{\alpha}}\right\}$.

The expression $\partial \xi^{k} / \partial \hat{x}^{\beta}$ can be obtained by employing the following relationship ( $X^{I}$ defines coordinates in Euclidean frame $E_{l}$ )

$\frac{\partial \hat{x}^{\beta}}{\partial \xi^{x}}=\frac{\partial \hat{x}^{\beta}}{\partial X^{I}} \frac{\partial X^{I}}{\partial \xi^{x}}=\hat{e}_{\beta} \cdot X_{, x^{*}}$

so that

$\left[\frac{\partial \xi^{\alpha}}{\partial \hat{x}^{\beta}}\right]^{t}=\left[\frac{\partial \hat{x}^{\beta}}{\partial \xi^{\alpha}}\right]^{-t}$

$\left[\frac{\partial \hat{x}^{\beta}}{\partial X^{l}}\right]$ is a $(2 \times 3)$ matrix of unit base vectors $\hat{e}_{\beta}$ (it is a part of orthogonal rotation matrix), while $\left[\frac{\partial X}{\partial \xi^{\alpha}}\right]$ is $(3 \times 2)$ matrix of the mid-surface components of base vectors $X_{, \alpha^{2}}(8)$. The shape functions derivatives can be derived with respect to the local Cartesian coordinates as

$\left\{N_{, \hat{x}^{\beta}}^{i}\right\}=\left[\frac{\partial \xi^{z}}{\partial \hat{x}^{\beta}}\right]^{t}\left\{\frac{\partial N^{I}}{\partial \xi^{\alpha}}\right\}=\left[\frac{\partial \hat{x}^{\beta}}{\partial \vec{c}^{x}}\right]^{-1}\left\{\frac{\partial N^{I}}{\partial \xi^{\alpha}}\right\}$,

$\left\{\begin{array}{l}N_{, \dot{x}}^{I} \\ N_{, \hat{y}}^{I}\end{array}\right\}=\left[\begin{array}{ll}\hat{e}_{1} \cdot X_{, \xi} & \hat{e}_{2} \cdot X_{, \xi} \\ \hat{e}_{1} \cdot X_{, \eta} & \hat{e}_{2} \cdot X_{, \eta}\end{array}\right]^{-1}\left\{\begin{array}{l}N_{, \xi}^{I} \\ N_{, \eta}^{I}\end{array}\right\}$.

In (46) (and in Appendix A, where finite element matrices are explicitly given) $(\bullet)_{{\dot{\dot{x}^{x}}}^{\underline{x}}}$ denotes the differentiation with respect to coordinate $\hat{x}^{x}$. As $\hat{x}^{1}$ and $\hat{x}^{2}$ are orthogonal, the mid-surface part of metric tensor now becomes an identity tensor $\left[A_{\alpha \beta \beta}\right]=\left[\delta_{\alpha \beta}\right]$.

The strain components are collected in the following strain vectors

$\varepsilon=\left\{\begin{array}{c}\varepsilon_{\dot{x} \dot{x}} \\ \varepsilon_{\hat{y} y} \\ 2 \varepsilon_{\hat{x} \hat{y}}\end{array}\right\}, \quad \kappa=\left\{\begin{array}{c}\kappa_{\dot{x} \hat{x}} \\ \kappa_{\hat{y} \hat{y}} \\ 2 \kappa_{\hat{x} \hat{y}}\end{array}\right\}, \quad \gamma=\left\{\begin{array}{l}\gamma_{\hat{x}} \\ \gamma_{\hat{y}}\end{array}\right\}$,

while the corresponding stress resultant vectors are

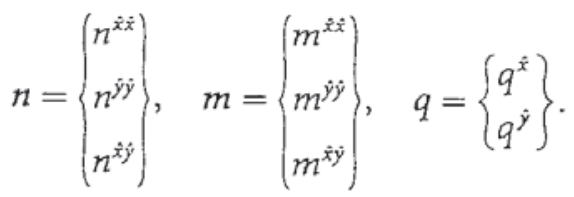

The finite element matrices are provided in Appendix A.

8.1

\section{Linear analysis results}

In this section some standard linear tests are presented in order to compare behaviour of the present finite element with some other four node elements recently reported in the literature.

Example I Shearing of the twisted beam. This often described problem is a benchmark test for the effect of warping. Finite element simulations result in significant errors if strain measures do not include coupling of curvatures with displacements (this is very often true for the resultant stress elements derived by degenerated continuum approach). The geometry and material characteristics are specified as following: The beam has a length of $L=12$, width $w=1.1$, thickness $t=0.05$, while the Young's modulus is $E=29.0 \times 10^{6}$, and the Poisson's ratio is $v=0.22$. In its initial configuration the beam has a $90^{\circ}$ twist (Fig. 3). Typically, two load cases are considered (see Fig. 3): (a) unit load at the beam end in the width direction and (b) unit load at the beam end in the thickness direction. From Fig. 4(a) - (b), which presents the test results, it may be observed that the overall accuracy of the element is in agreement with accuracy of test results presented by Parisch (1991) and by Simo et al. (1989b).

Example 2 Pinched cylinder with end diaphragms. This problem is regarded as a challenging linear test for shell elements, since both the inextensional deformations and complex state of membrane stresses, significantly influence the shell behaviour under loading. The geometry and material characteristics for this problem are specified as following: The length of the cylinder is $L=600$, radius is $R=300$ and thickness is $t=3$ (Fig. 5), while the Young's modulus is $E=3.0 \times 10^{6}$ and the Poisson's ratio is $v=0.3$. A force $F=1.0 / 4$ is applied on one octant of the shell while the appropriate symmetry conditions (geometry and loading) are imposed. The results are shown in Fig. 6 and are normalised against the analytically obtained value of $1.82488 \times 10^{-5}$ given by Simo et al. (1989b). The results for some representative shell elements are also presented. In Fig. 6 '4-DKQ' stands for a discrete Kirchhoff quadrilateral (its results are taken from Simo et al. 1989b). It can be observed that all elements, except 4-DKQ, behave almost identically with results falling within $93 \%-95 \%$ (for mesh $16 \times 16$ ) of the

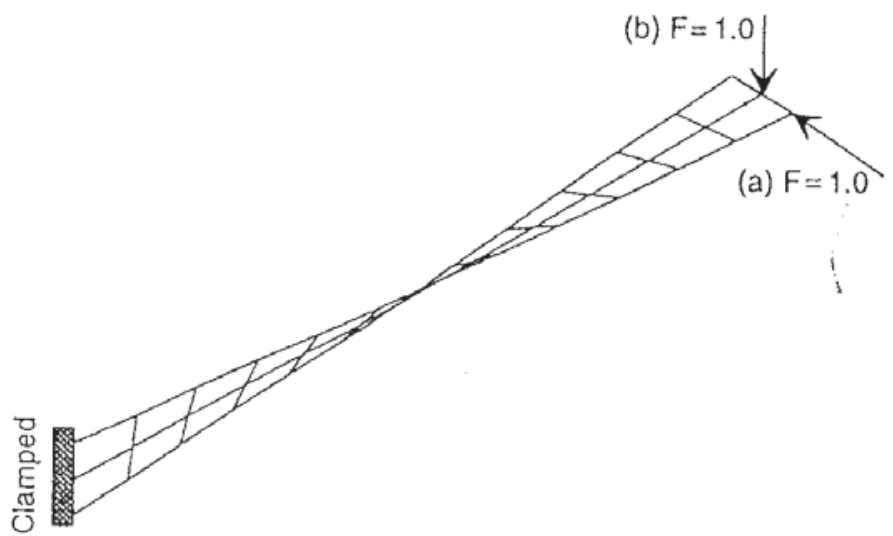

Fig. 3. Twisted beam: Geometry and loading 


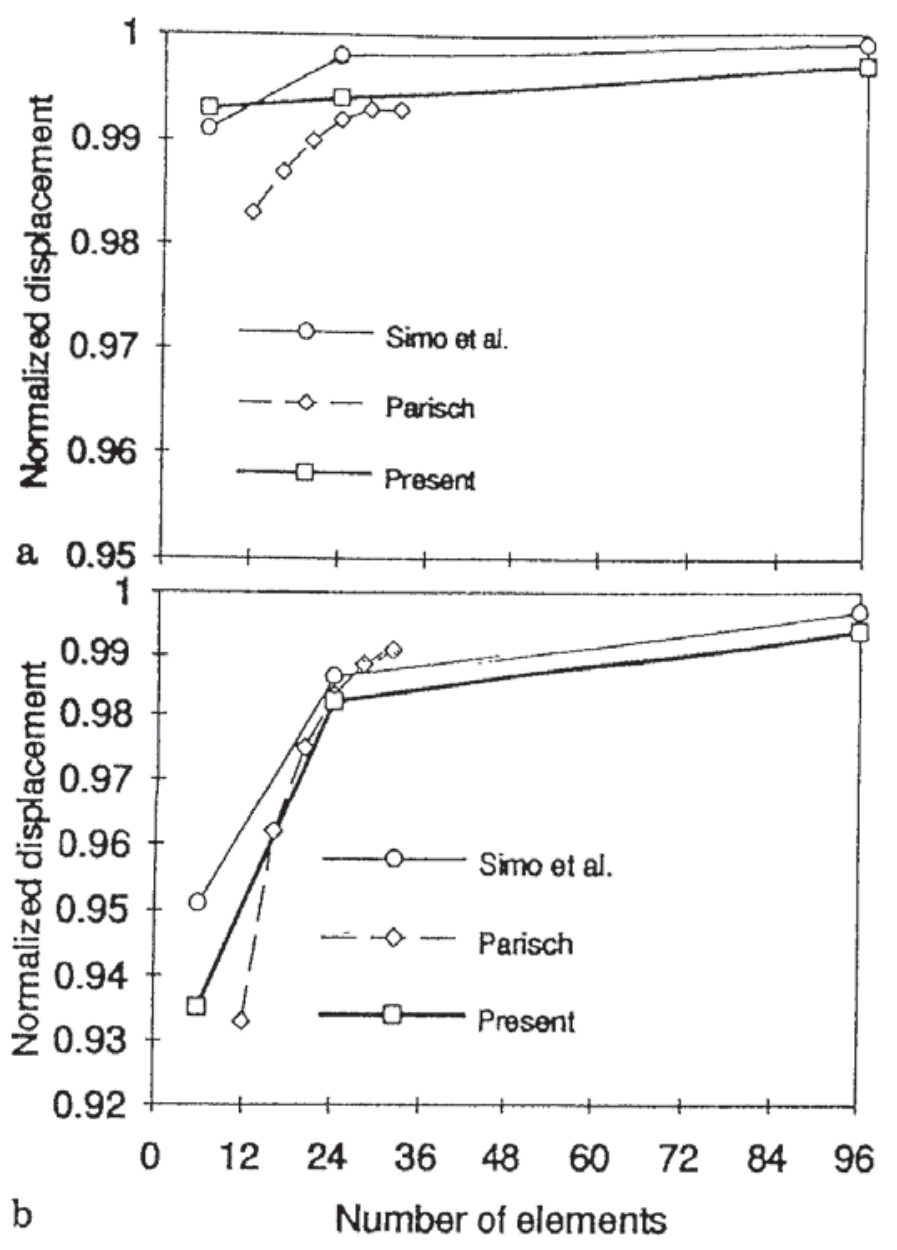

Fig. $4 a, b$. Twisted beam: a Displacement under the force, load case (a). b Displacement under the force, load case (b)

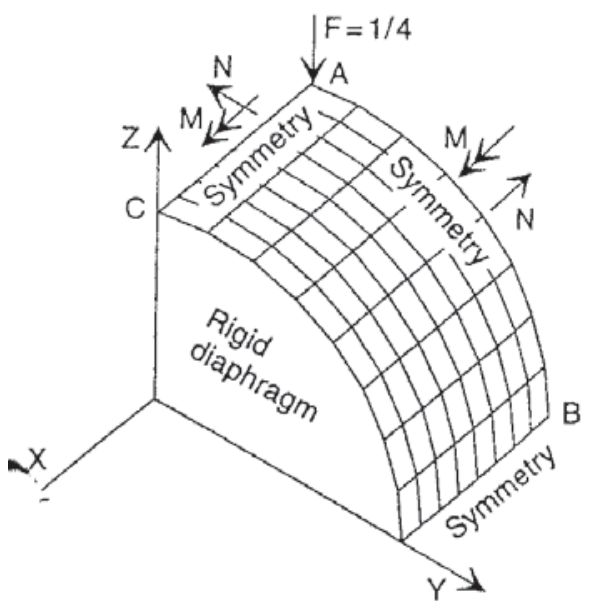

Fig. 5. Pinched cylinder: Geometry and loading

analytical value. This observation applies also for the mixed four node element proposed by Simo et al. (1989b) (denoted by $-\mathrm{M}$ in Fig. 6), which usually shows a superior performance when compared to displacement based elements in membrane dominated problems.

To illustrate the accuracy of solution, the stress resultants (i.e. normal components of membrane force and bending moment) distribution along the curves $\mathrm{AB}$ and $\mathrm{AC}$ of the cylinder (see Fig. 5) are compared in Fig. 7 with the analytical solutions obtained by Lindberg et al. (1969) using the Fourier series expansion. The correspondence between the finite element results and the analytical solution is excellent even in

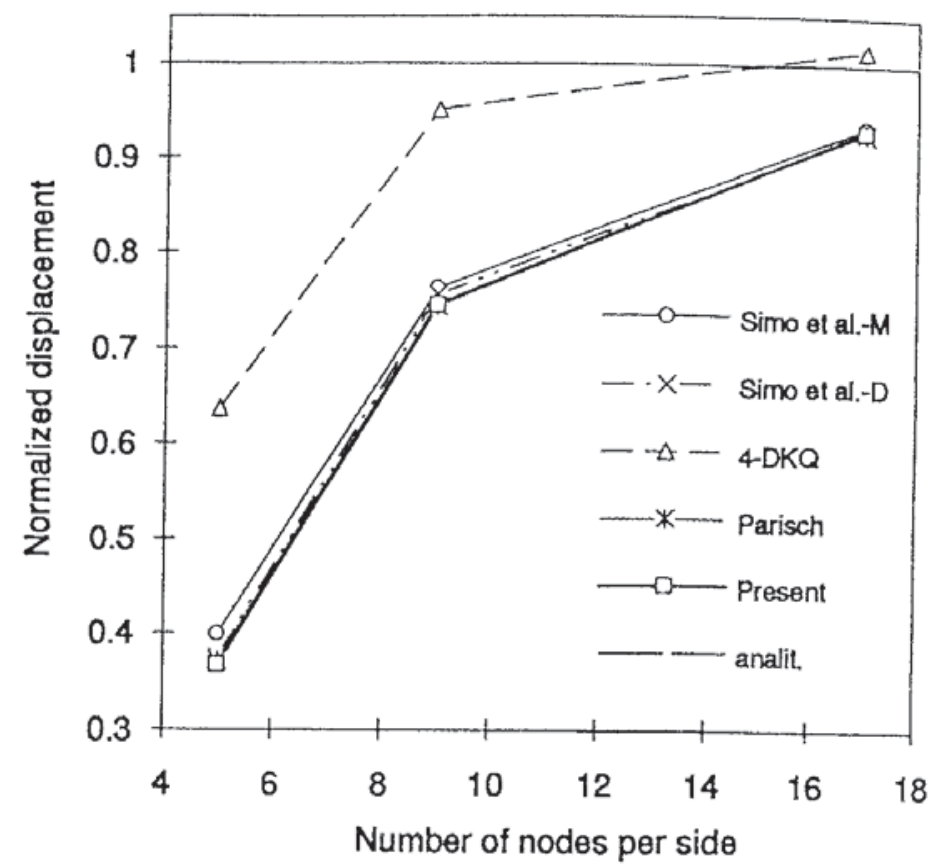

Fig. 6. Pinched cylinder: Displacement under the force

the vicinity of the point load.

Example 3 Pinched hemispherical shell. The shell with radius $R=10$, thickness $t=0.04$ (Fig. 8), and elastic material properties $E=6.825 \times 10^{7}$ and $v=0.3$, is fixed at the pole, $X=R$, and pinched by four forces of $F=2 P=2.0$. By employing symmetry conditions only one quadrant is considered in the finite element simulations. The results, normalised to the analytically obtained value of 0.0924 (Simo et al. 1989b) are presented in Fig. 9 and compared to some other published solutions. In Fig. 9 'RSDS' stands for the four node resultant stress degenerated shell element with reduced integration and stabilisation (the results are taken from Simo et al. 1989b). It can be seen that the present element behaves slightly stiffer when compared to the mixed formulation based element (Simo et al. 1989b) and provides similar response to Parisch (1991).

Example 4.1 Mesh distortion - Simply supported square plate under central point load. To test sensitivity of the mesh distortion on quality of finite element results two standard tests have been performed. In the first test, a quarter of the simply supported plate under central point load is gradually distorted by moving the middle node of the mesh, as shown in Fig. 10. In Table 1 results of the finite element calculation are expressed in terms of the ratio to the analytical solution (Timoshenko and Woinowsky-Krieger, 1959) for plate thicknesses $t=0.02$ and $t=0.004$. The performance of the present shell element is very good even in situations when element becomes highly distorted and exhibits a negative Jacobian.

Example 4.2 Mesh distortion-Bending of a clamped beam. In the second mesh distortion test a clamped beam is, at its free end, loaded by three different loading conditions. The length of the beam is $L=10 \mathrm{~m}$, width is $b=1 \mathrm{~m}$ while height is $h=1 \mathrm{~m}$. The Young's modulus and Poisson's ratio are chosen as $E=10^{7}$ and $\nu=0.3$, respectively. The regular and distorted mesh are shown in Fig. 11 together with loading conditions. 

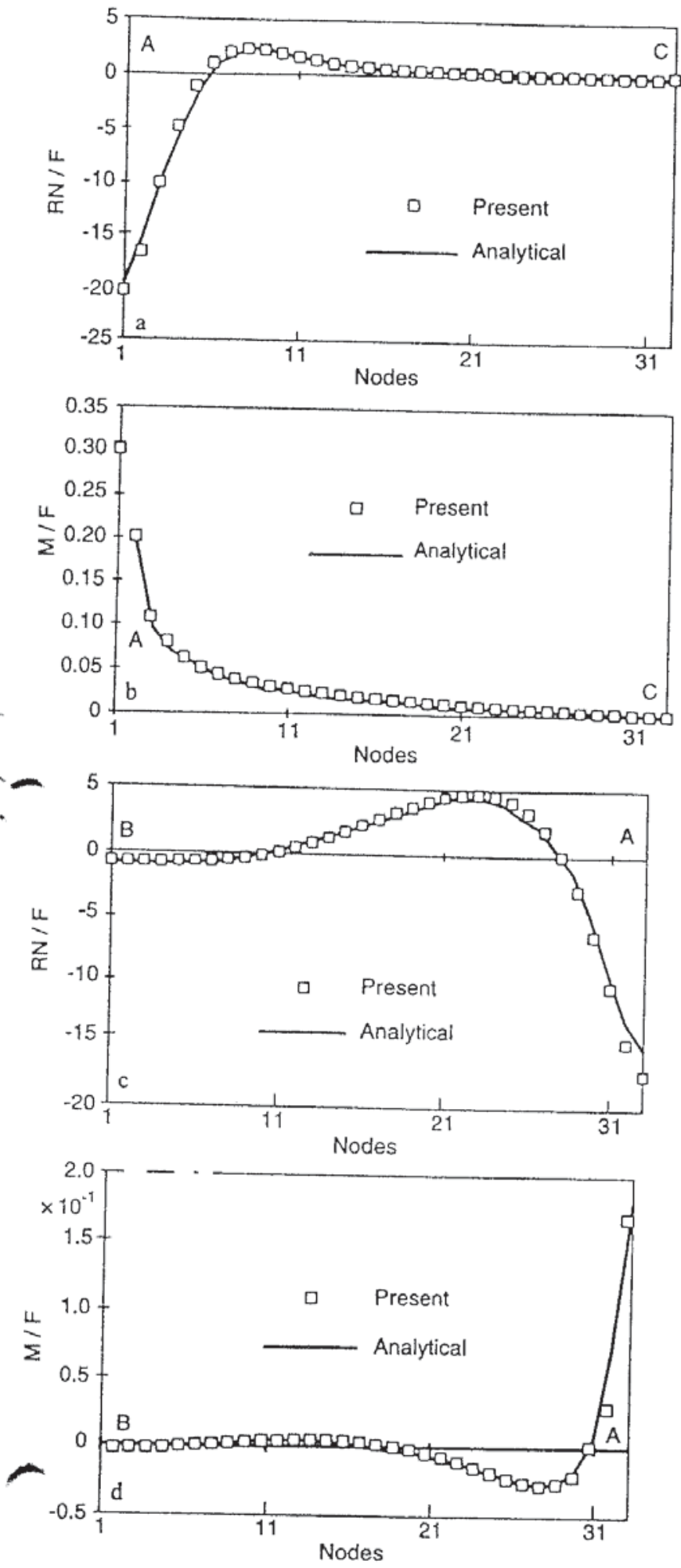

Fig. 7a d. Pinched cylinder: Stress resultant distributions. $a-b$ Membrane force and bending moment distributions along the line AC. $c-d$ Membrane force and bending moment distributions along the curve $A B$

In Table 2 the results obtained by finite element calculations are compared with analytical solutions based on the beam theory. Clearly, results confirm a very good performance of the present element with a small degree of mesh sensitivity in the case of complex torsional loading condition.

\section{2}

\section{Non-linear analysis results}

In this section non-linear numerical examples are considered to illustrate the performance of the presented element on a range

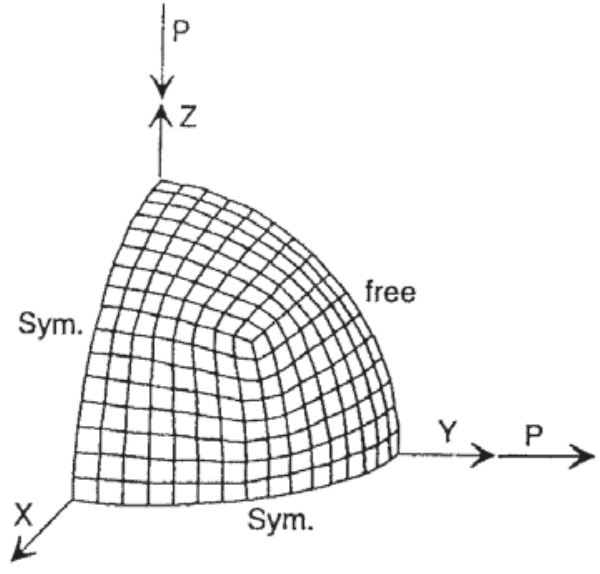

Fig. 8. Hemispherical shell: Geometry and loading

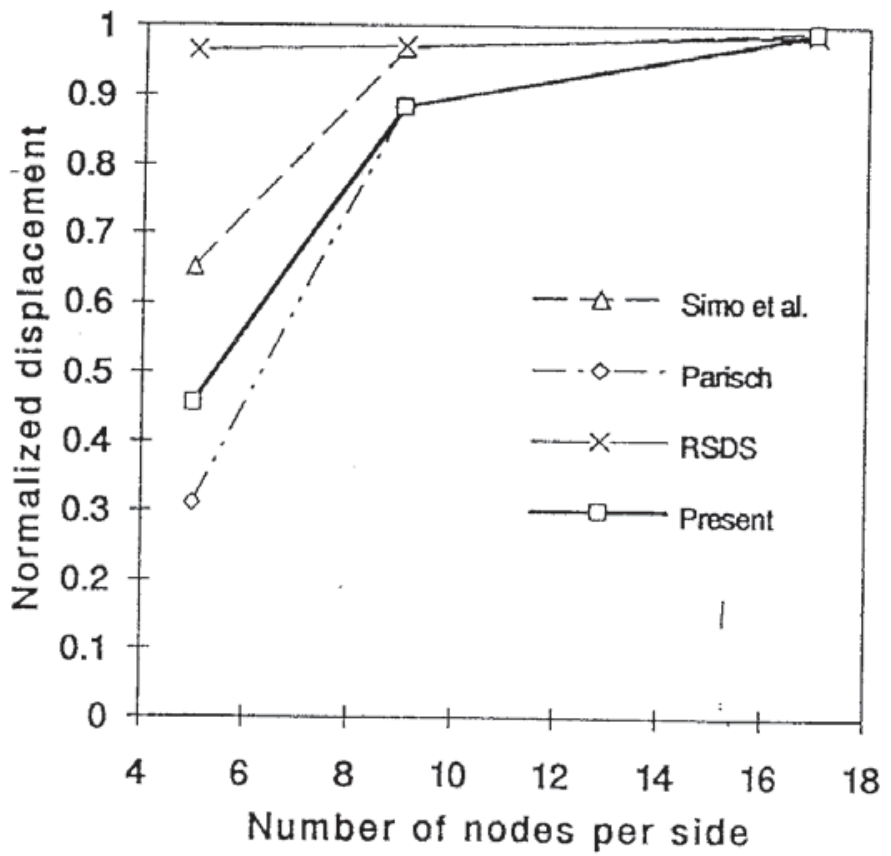

Fig. 9. Hemispherical shell: Displacements under the forces

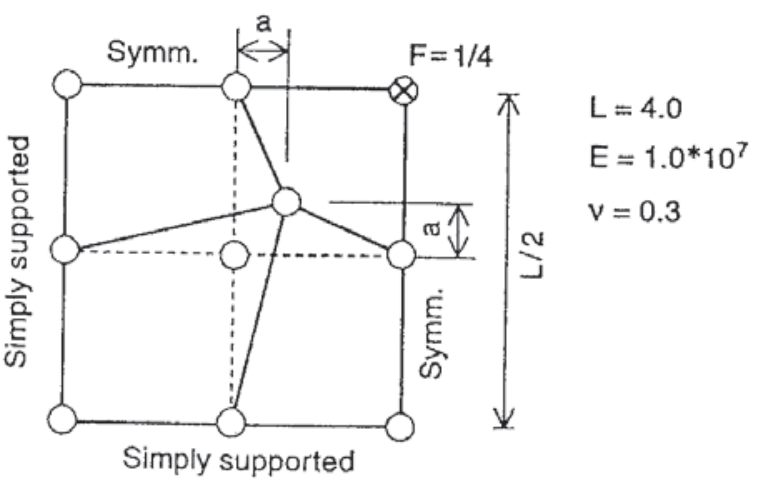

Fig. 10. Mesh distortion - Simply supported plate under central point load: Geometry and material characteristics

of nonlinear tests. A comparison is provided with the results reported in the literature.

In all examples the full Newton-Raphson method is used, with a tangent stiffness arising from the consistent linearisation of the adopted shell model. Convergence of the finite element solution is established on the basis of the standard Euclidian norm of the out-of-balance forces. The use of consistent tangent 

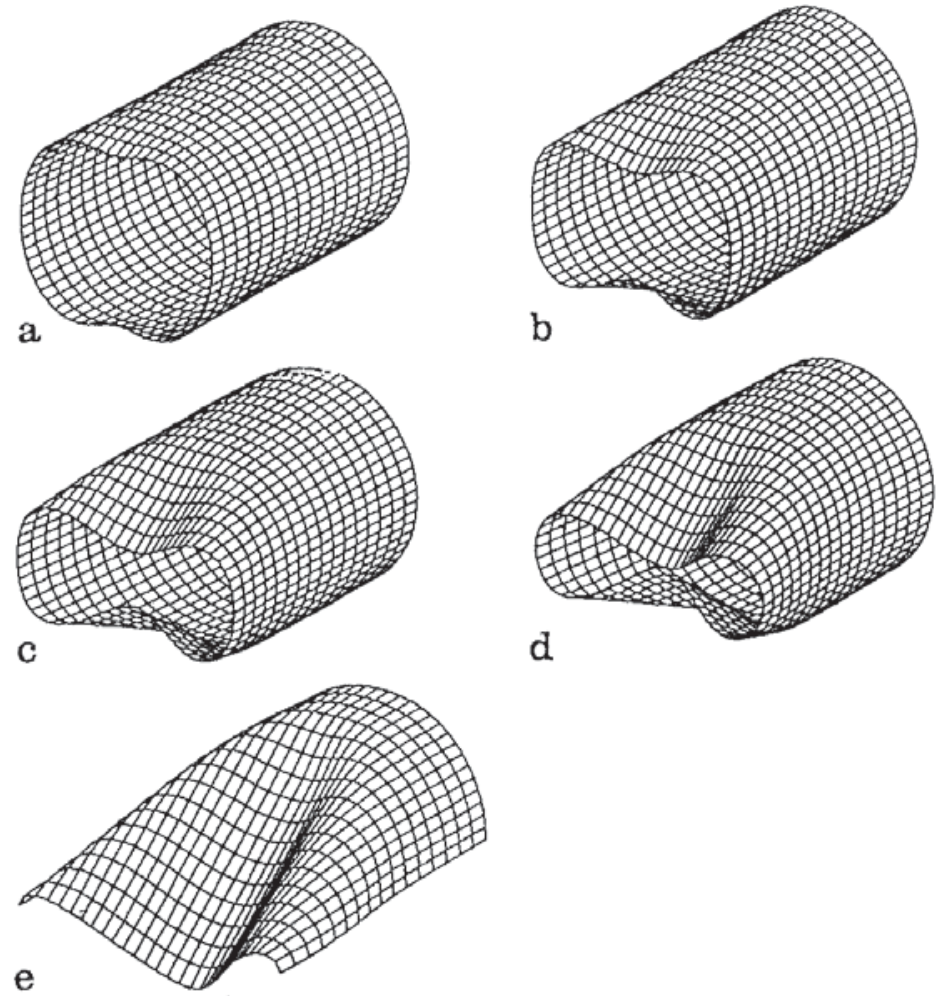

d

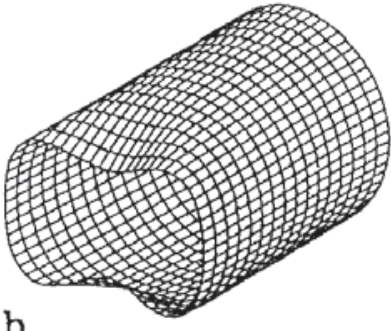

Fig. 14a-e. Clamped cylinder: Deformed configuration of the shell at 5 different load stages. Displacements under the forces are: $\mathrm{a} R / 4, \mathrm{~b} R / 2$, c $3 R / 4, \mathrm{~d} R$ and e $1.608 R$

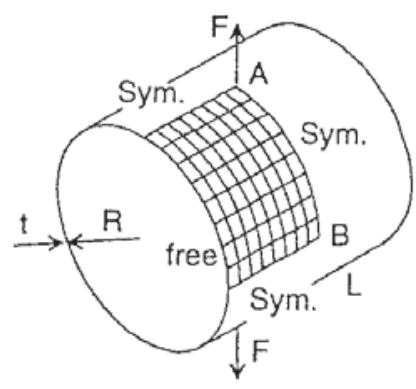

Fig. 15. Cylinder with free ends: Geometry and loading

and Sansour and Bufler (1992). Using different finite elements and different meshes they arrived to the solutions that slightly distinguish from each other. Three different meshes $8 \times 8$, $16 \times 16$ and $32 \times 32$ are considered in the present analysis. Computation is performed by load control and the size of the increments is set to be very small at the region where snap-through is observed Perić and Owen (1991). The standard check of the residual forces is performed with convergence tolerance set to $1.0 \times 10^{-7}$.

The response of the shell can be divided into two regions: the first one is characterised by large displacements and low (bending) stiffness and the second one is characterised by very stiff response of the shell. Force $F$ versus displacement curves of points A and B are presented in Figs. 16 and 17. Solutions obtained with $16 \times 16$ and $32 \times 32$ meshes (which practically coincide) are in very good agreement with the solutions obtained by Sansour and Bufler (1992) who used 9 node element (denoted as 9-NE). It is interesting to note that constant strain - constant curvature triangular Morley element (see Perić and Owen 1991) gives more flexible results. In Fig. 18 four different equilibrium configurations are depicted.

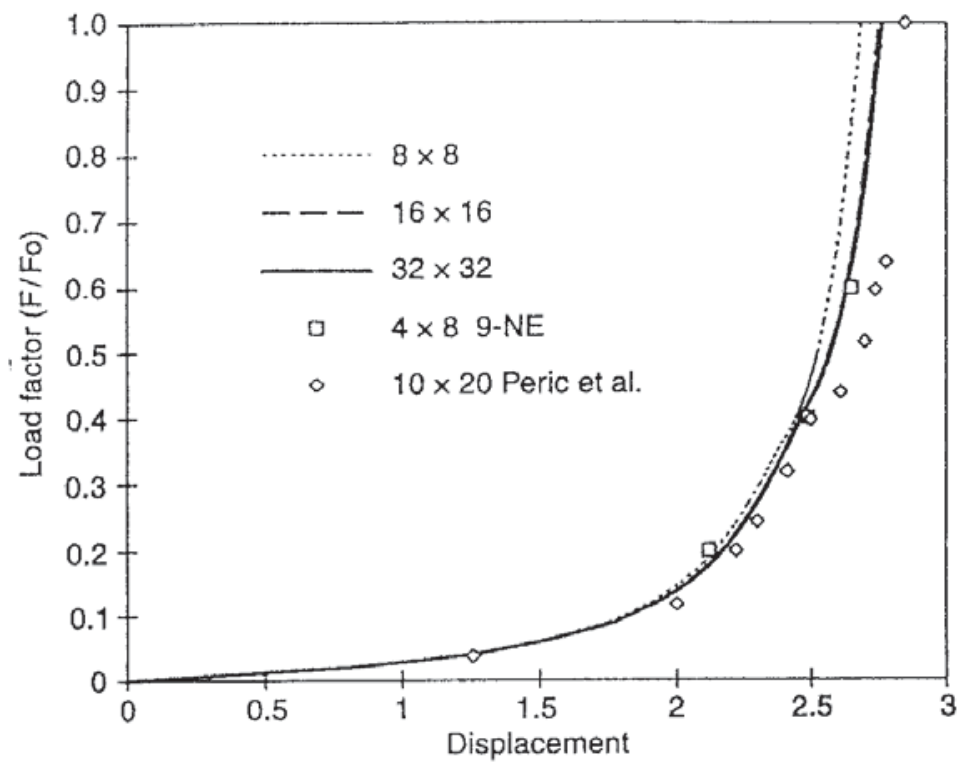

Fig. 16. Cylinder with free ends: Load versus displacement curves for point $A$

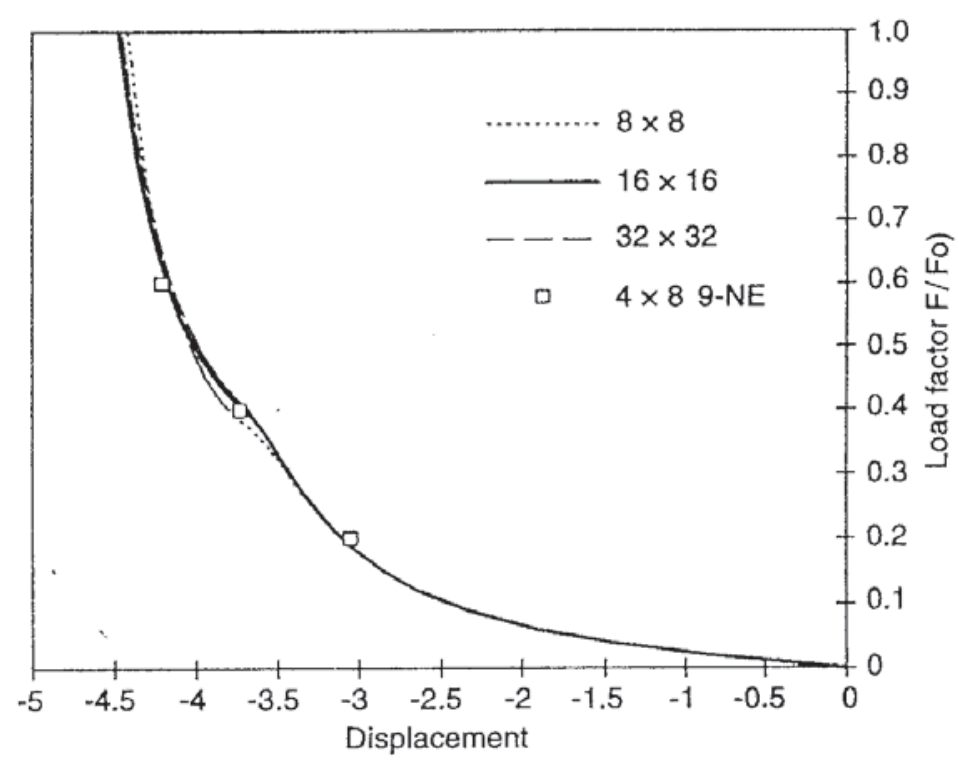

Fig. 17. Cylinder with free ends: Load versus displacement curves for point $B$

Results for the Euclidian norm of residuals $\|G\|$ given in Table 3 clearly illustrate quadratic rate of asymptotic convergence typical for the Newton-Raphson based iterative procedure.

Example 7 Ring plate loaded at the free edge. The ring plate is clamped at one end and subjected to a line dead load $p=0.1 * f$ at the other end. The geometry and loading conditions are shown in Fig. 19. The thickness of the plate is $t=0.03$. The elastic material properties are $E=2.1 \times 10^{8}$ and $v=0.0$. This example was considered by Büchter and Ramm (1992) and Wriggers and Gruttmann (1993), among others. In present simulation the plate is discretised with $8 \times 48$ and $4 \times 32$ four node elements. The 'cylindrical' arc-length method (Crisfield 1991) is used to follow the equilibrium path. The standard check of the residual forces was performed with convergence tolerance set to $1.0 \times 10^{-5}$. The load versus displacement curves for three characteristic points are depicted in Fig. 20. The maximum load factor for the coarsest mesh is 


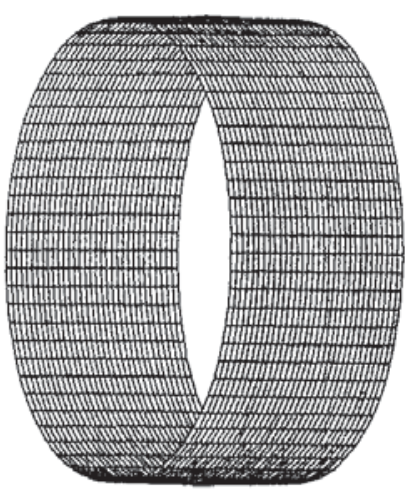

a

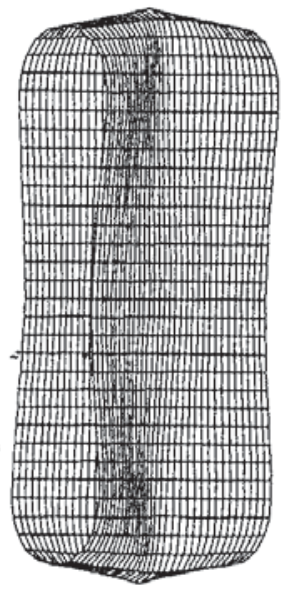

c

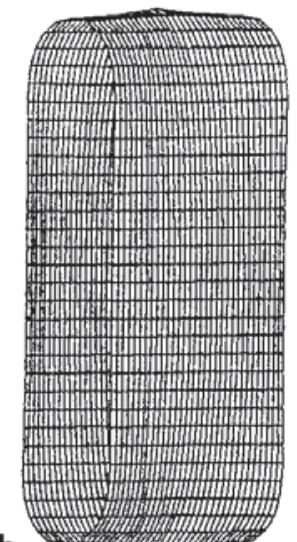

b

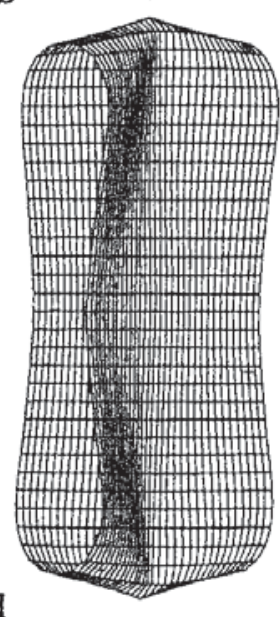

Fig. 18a-d. Cylinder with free ends: Deformed finite element mesh $(16 \times 16)$ at various stages of loading: a $F / F_{0}=0.04, \mathrm{~b} F / F_{0}=0.33$, c $F / F_{0}=0.60, \mathrm{~d} F / F_{0}=1.0$

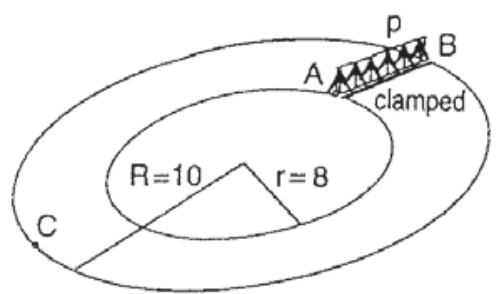

Fig. 19. Ring plate: Geometry and loading

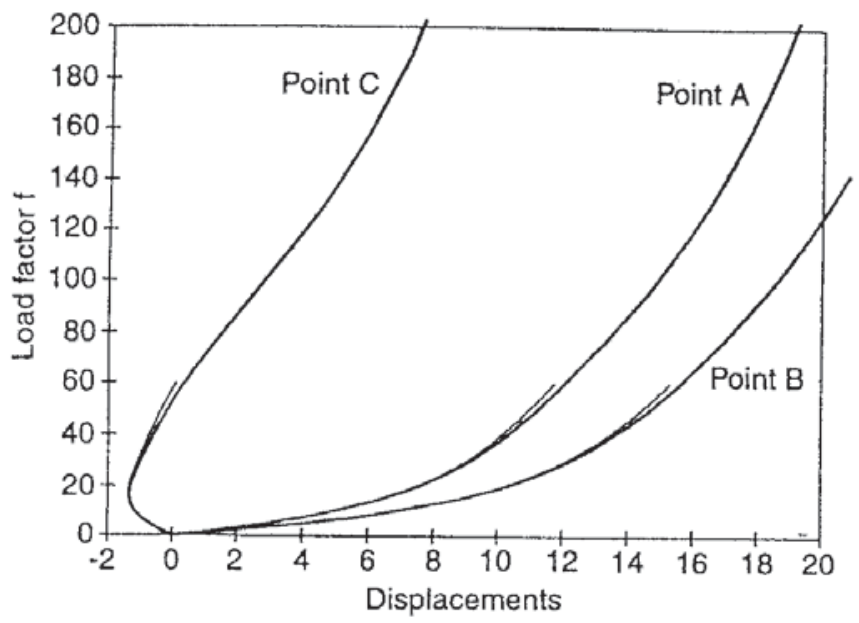

Fig. 20. Ring plate: Load versus displacement curves for points $A, B$ and $\mathrm{C}$

set to 60 (thin line). With the finer mesh the analysis is performed up to $f=202.53$ (thick line). Although not drawn in the figure, the agreement with the solutions by Büchter and Ramm (1992) and by Wriggers and Gruttmann (1993) is very good for the finer mesh, while the coarser mesh tends to

\begin{tabular}{lllll}
\hline Iteration & $F / F_{0}=0.0 \cdots 0.0774$ & $F / F_{0}=0.0774-0.3089$ & & $F / F_{0}=0.3089-0.7358$ \\
\cline { 1 - 1 } 2 & $0.1919 \times 10^{3}$ & $0.7255 \times 10^{2}$ & $0.6747 \times 10^{2}$ \\
3 & $0.9511 \times 10^{2}$ & $0.5368 \times 10^{1}$ & $0.4860 \times 10^{1}$ \\
4 & $0.2827 \times 10^{2}$ & $0.3523 \times 10^{2}$ & $0.1665 \times 10^{2}$ \\
5 & $0.9491 \times 10^{1}$ & $0.2499 \times 10^{1}$ & $0.6554 \times 10^{0}$ \\
6 & $0.6952 \times 10^{1}$ & $0.4093 \times 10^{1}$ & $0.5261 \times 10^{0}$ \\
7 & $0.5919 \times 10^{0}$ & $0.1346 \times 10^{0}$ & $0.2835 \times 10^{-2}$ \\
8 & $0.2733 \times 10^{0}$ & $0.5483 \times 10^{-1}$ & $0.1403 \times 10^{-3}$ \\
9 & $0.8981 \times 10^{-3}$ & $0.2249 \times 10^{-2}$ & $0.1766 \times 10^{-9}$ \\
\hline & $0.1224 \times 10^{-5}$ & $0.9416 \times 10^{-5}$ & \\
\hline
\end{tabular}

Table 3. Stretching of a cylinder with free ends: Euclidian norms of residuals for three typical load steps for $16 \times 16$ mesh

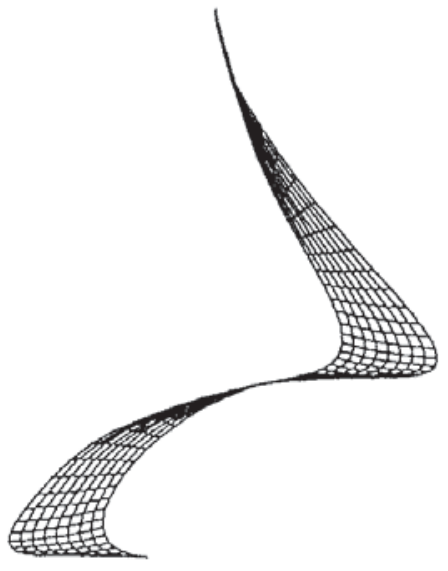

d b

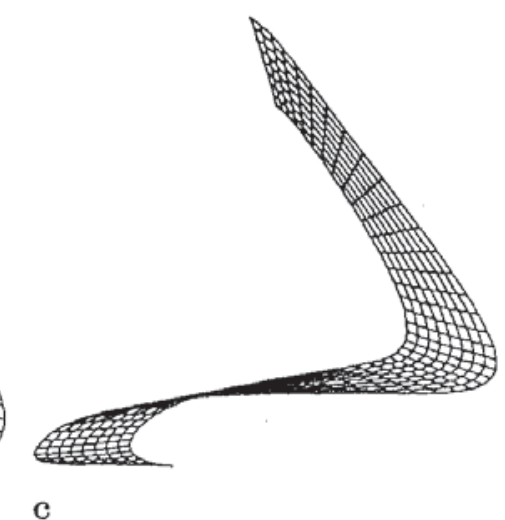

c

Fig. 2la-d. Ring plate: Deformed finite element mesh $(8 \times 48)$ at different loading stages: $\mathbf{a} f=11.726, \mathrm{~b} f=53.786, \mathrm{c} f=105.55, \mathrm{~d} f=202.53$ 
Table 4. Ring plate loaded at the free edge: Euclidian norms of residuals for two typical load steps for $4 \times 32$ mesh

\begin{tabular}{|c|c|c|}
\hline Iteration & $f=7.152-7.580$ & $f=20.535-21.360$ \\
\hline 1 & $0.1222 \times 10^{5}$ & $0.3816 \times 10^{5}$ \\
\hline 2 & $0.2016 \times 10^{1}$ & $0.7391 \times 10^{0}$ \\
\hline 3 & $0.2708 \times 10^{1}$ & $0.1394 \times 10^{1}$ \\
\hline 4 & $0.1226 \times 10^{-1}$ & $0.8570 \times 10^{-3}$ \\
\hline 5 & $0.4453 \times 10^{-2}$ & $0.3804 \times 10^{-5}$ \\
\hline 6 & $0.1590 \times 10^{-6}$ & \\
\hline
\end{tabular}

behave slightly stiffer. In Fig. 21 four deformed configurations of ring plate are presented.

Results for the Euclidian norm of residuals $\|G\|$ provided in Table 4 for mesh $4 \times 32$ again illustrate quadratic rate of asymptotic convergence.

Example 8. Composite shallow cylindrical shell with a central point load. Saigal et al. (1986) performed numerical tests on composite shallow cylindrical shell with a point load at its centre. The same shallow shell was later considered by Laschet and Jeusette (1990). A similar geometry is often used to study performance of finite element models and solution techniques in the nonlinear range. Geometry and loading conditions of the shell are shown in Fig. 22. The shell is simply supported along its straight edges (all translational displacements are fixed while both rotations are allowed) and free and its curved edges. The geometric data used in the analysis is as follows (Saigal et al. 1986): The legth of the panel is $L=508 \mathrm{~mm}$, radius is $R=2540 \mathrm{~mm}$, while the curved edge is defined by the angle $\theta=0.2 \mathrm{rad}$ which gives the arc-length of its curved edges $S=508 \mathrm{~mm}$. Following standard practice two panel thicknesses are chosen: $t_{A}=12.6 \mathrm{~mm}$ and $t_{B}=6.3 \mathrm{~mm}$. The panel is built up by a lay-up of 12 plies with a ply thickness equal to $t_{A} / 12=$ $1.05 \mathrm{~mm}$ and $t_{B} / 12=0.525 \mathrm{~mm}$ for the panel $\mathrm{A}$ and $\mathrm{B}$, respectively. To investigate the effects of lay-up on performance two choices for the ply lay-up have been considered: (i) $\left[90_{4}^{\circ} / 0_{4}^{\circ} / 90_{4}^{\circ}\right]$ and (ii) $\left[0_{4}^{\circ} / 90_{4}^{\circ} / 0_{4}^{\circ}\right]$. The angle, describing ply fibre orientations, is measured between the fibre direction and projection of the axis $X_{2}$ to the shell surface. The material characteristics of the carbon-epoxy layers are as follows

(Laschet and Jeusette 1990): the elastic moduli are $E_{1}=3.3 \mathrm{kN} / \mathrm{mm}^{2}, E_{2}=E_{3}=1.1 \mathrm{kN} / \mathrm{mm}^{2}$, while the in-plane shear modulus is $G_{12}=0.66 \mathrm{kN} / \mathrm{mm}^{2}$ and Poisson's ratio is

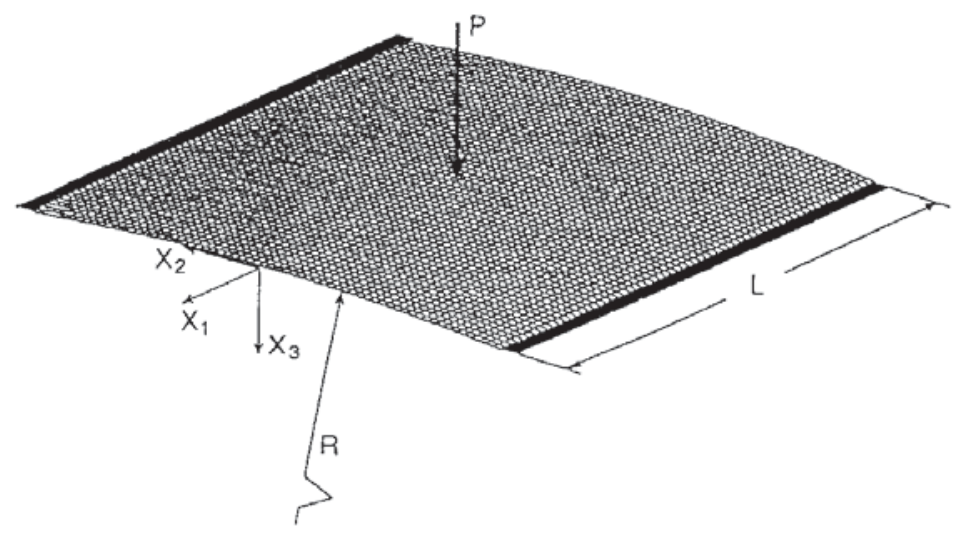

Fig. 22. Composite cylindrical shell: Geometry and loading $v_{12}=0.25$. Additionaly, the values of the transverse shear moduli are taken as $G_{13}=G_{23}=0.66 \mathrm{kN} / \mathrm{mm}^{2}$, while the transverse shear factor is evaluated with accordance to the procedure provided by Figueiras and Owen (1984).

In the present analysis, due to symmetry of the problem only quarter of the panel is considered, with appropriate boundary conditions imposed. A finite element simulation is performed with a mesh containing $32 \times 32$ four-node elements. A

'cylindrical' arc-length method is used to follow the path of equilibrium configurations.

The central point load versus vertical displacement diagram are shown in Figs. 23 and 24 for the panels A and B, respectively. In the case of thicker panel A results exhibit standard limit load instability type behaviour and compare well with previously published results (Laschet and Jeusette 1990, Saigal et al. 1986). As expected, the second lay-up $\left[0_{4}^{\circ} / 90_{4}^{\circ} / 0_{4}^{\circ}\right]$ shows a significantly stiffer response to the prescribed loading conditions. Results for the thinner panel B, however, show some interesting

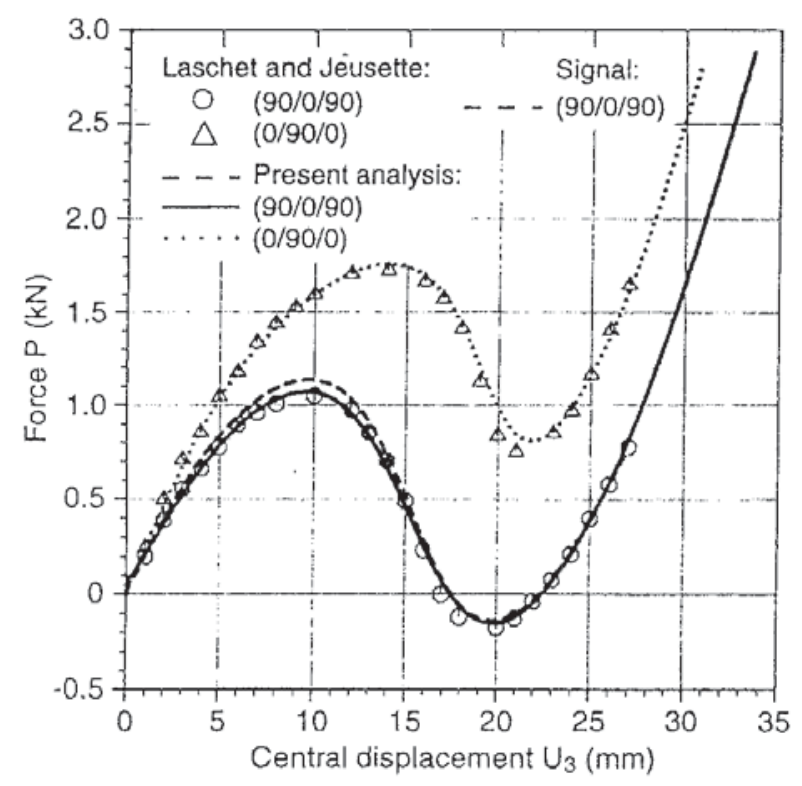

Fig. 23. Composite cylindrical shell: Central point load versus displacement diagram for the panel A with thickness $t_{A}=12.6 \mathrm{~mm}$

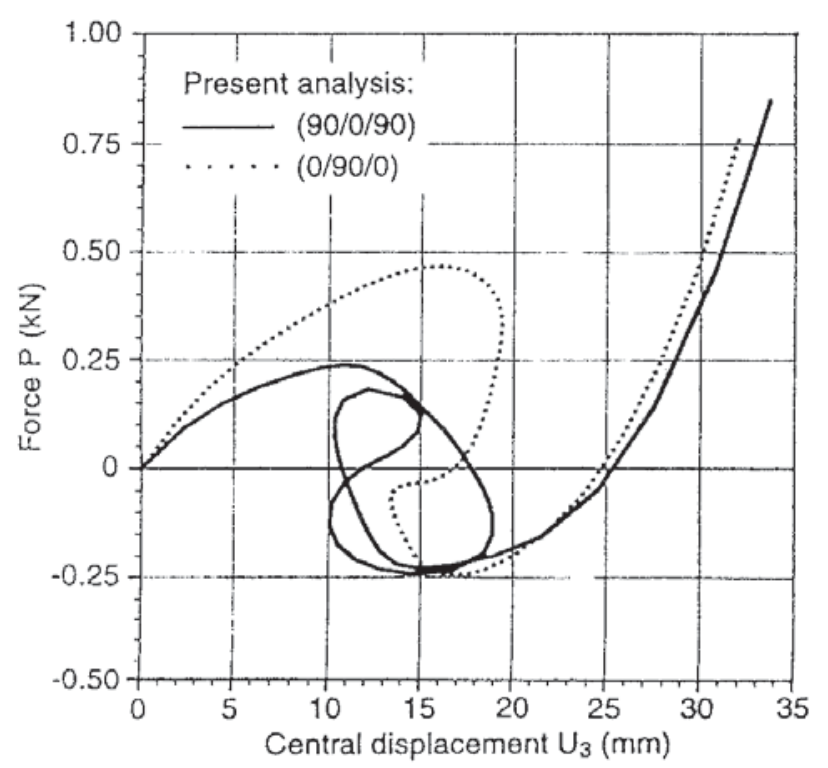

Fig. 24. Composite cylindrical shell: Central point load versus displacement diagram for the panel B with thickness $t_{B}=6.3 \mathrm{~mm}$ 

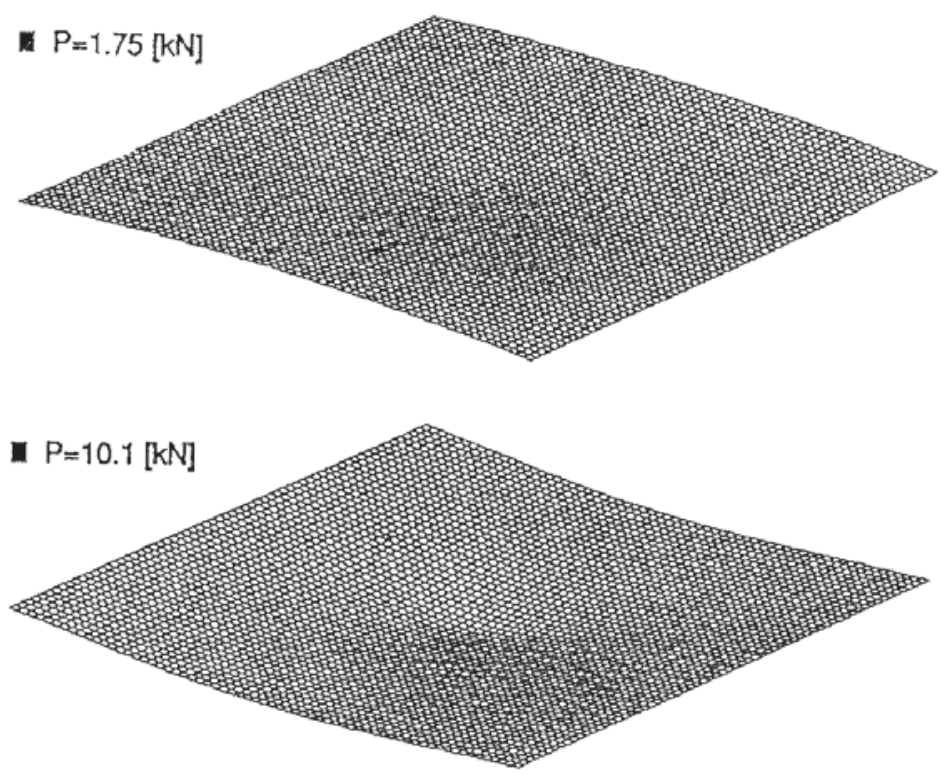

Fig. 25. Composite cylindrical shell: Deformed finite element meshes

features. While for the second lay-up a loading-displacement urve have been obtained which is typical for this problem, with a clear snap-back behaviour, the first lay-up $\left[90_{4}^{\circ} / 0_{4}^{\circ} / 90_{4}^{\circ}\right]$ produces unexpected and very rich equilibrium path structure. The equilibrium path exhibits several limit points before reaching its stable stiff equilibrium branch corresponding to a simple configuration with a dominant tensile action. Although of some interest for this example, issues related to bifurcation analysis, branch-switching techniques and determination of the singular points, are not the objective of this work and will not be further detailed in this paper (for recent discussion on computational aspects of bifurcation analysis we refer to Wriggers and Simo (1990)).

Figure 25 depicts the deformed finite element meshes of the panel A for the second ply lay-up at central point load of $P=1.75 \mathrm{kN}$ and $P=10.1 \mathrm{kN}$. The corresponding central point displacements are $U_{3}=13.79 \mathrm{~mm}$ under maximum limit load and $U_{3}=42.64 \mathrm{~mm}$ on the stable equilibrium branch.

In agreement with all previous examples presented in this section, the consistent linearisation of the shell kinematics, results in the Newton-Raphson based iterative numerical iocedure, which preserves quadratic rate of asymptotic convergence. As an illustration, results for the Euclidian norm of residuals $\|G\|$ are provided in Table 5 for three typical load steps for the panel with thickness $t_{A}=12.6 \mathrm{~mm}$ and lay-up $\left[0_{4}^{\circ} / 90_{4}^{\circ} / 0_{4}^{\circ}\right]$.

Table 5. Composite shallow cylindrical shell with a central point load: Euclidian norms of residuals for three typical load steps for the panel with thickness $t_{A}=12.6 \mathrm{~mm}$ and lay-up $\left[0_{4}^{\circ} / 90_{4}^{\circ} / 0_{4}^{\circ}\right]$

\begin{tabular}{|c|c|c|c|}
\hline Iteration & $P=1.318-1.606$ & $P=1.644-1.465$ & $P=1.746-2.825$ \\
\hline 1 & $0.5714 \times 10^{-1}$ & $0.2772 \times 10^{-1}$ & $0.7329 \times 10^{-1}$ \\
\hline 2 & $0.8655 \times 10^{-3}$ & $0.2277 \times 10^{-2}$ & $0.5909 \times 10^{-3}$ \\
\hline 3 & $0.4359 \times 10^{-6}$ & $0.1485 \times 10^{-5}$ & $0.1453 \times 10^{-8}$ \\
\hline 4 & $0.2692 \times 10^{-10}$ & $0.2884 \times 10^{-10}$ & \\
\hline
\end{tabular}

\section{Conclusion}

Some computational aspects of thin elastic shells undergoing large rotations are presented and applied in finite element formulation of a simple four node isoparametric stress resultant shell element. Variable material properties are incorporated in a model, by performing an analytical integration across the thickness. The resulting model allows for simulation of a general elastic material with varied degree of orthotropy, which is typical for fibre reinforced composites. Thus a simple and efficient model suitable for analysis of multilayered composite shells is attained.

Large rotations are parameterized with two Euler angles which has proved robust and efficient for the present applications. Consistent linearisation of the adopted shell model is performed, leading to an effective numerical algorithm, which exhibits a quadratic rate of asymptotic convergence. Local Cartesian frames are defined at numerical integration points in order to simplify the governing expressions and prepare the formulation to include inelastic material models.

Throughout the formulation the elastic strains are restricted to be small. The small strain elasto-plastic shell model, along with the formulation for the large rotations which does not posses singularities, will be considered in the subsequent report.

Using the formulation presented, some linear and nonlinear tests have been performed. The results are in close agreement with those reported in recent publications.

Finally, it should be emphasised that the presented approach may be applicable to laminated composite shells within the elastic regime for ply stiffnesses and orientations whcih are not too dissimilar. However, the complete treatment of laminated composites under inelastic deformation, incorporating damage and/or elasto-plastic material behaviour, and more importantly including delamination effects, necessitates the consideration of higher order or local theories in which the variation of in-plane deformation through the laminate thickness is taken into account.

\section{Appendix A}

\section{A.1}

\section{Isotropic constitutive equations}

The standard constitutive relations in the local Cartesian frame are obtained from (22) and (23). The membrane, bending and transverse shear parts are given as follows

$$
\begin{aligned}
& H_{m}=\frac{E h}{1-v^{2}}\left[\begin{array}{ccc}
1 & v & 0 \\
v & 1 & 0 \\
0 & 0 & \frac{1-v}{2}
\end{array}\right]_{(3 \times 3)}, \\
& H_{b}=\frac{E h^{3}}{12\left(1-v^{2}\right)}\left[\begin{array}{ccc}
1 & v & 0 \\
v & 1 & 0 \\
0 & 0 & \frac{1-v}{2}
\end{array}\right]_{(3 \times 3)}, \\
& H_{s}=\kappa G h\left[\begin{array}{ll}
1 & 0 \\
0 & 1
\end{array}\right]_{(2 \times 2)}
\end{aligned}
$$


The complete constitutive matrix $\mathrm{H}$ then may be represented in the block-diagonal matrix form as

$\mathrm{H}=\operatorname{diag}\left[\mathrm{H}_{m}, \mathrm{H}_{b}, \mathrm{H}_{s}\right]_{(8 \times 8)}$,

Clearly, the matrix $\mathrm{H}$ relates (47) and (48) as

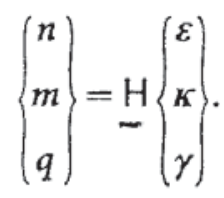

\section{A.2}

\section{Discrete strain-displacement operator matrix}

Starting from (21), and using (24), (27), (28) and (46), the following discrete strain-displacement operator matrix relative to the local Cartesian frame is obtained

$$
\left\{\begin{array}{l}
\delta \varepsilon \\
\delta \kappa \\
\delta \gamma
\end{array}\right\}_{(8 \times 1)}=\left[\begin{array}{llll}
B_{m}^{1} & B_{m}^{2} & B_{m}^{3} & B_{m}^{4} \\
B_{b}^{1} & B_{b}^{2} & B_{b}^{3} & B_{b}^{4} \\
B_{s}^{1} & B_{s}^{2} & B_{s}^{3} & B_{s}^{4}
\end{array}\right]_{(8 \times 20)}\left(\begin{array}{c}
\delta r_{1} \\
\delta r_{2} \\
\delta r_{3} \\
\delta r_{4}
\end{array}\right\}_{(20 \times 1)},
$$

where $\delta r_{I}$ are virtual displacements and 'rotations' at the node ' $I$ ' $(I=1,2,3,4)$. The membrane part of the discrete strain-displacement operator related to the node ' $I$ ', $B_{m}^{I}$ ' may be expressed as

$$
B_{m}^{I}=\left[\begin{array}{cc}
N_{, \hat{x}}^{I}\left(x_{, \hat{x}}\right)^{t} & 0 \\
N_{, \hat{y}}^{I}\left(x_{, \hat{y}}\right)^{t} & 0 \\
N_{, \hat{x}}^{I}\left(x_{, \hat{y}}\right)^{t}+N_{, \hat{y}}^{I}\left(x_{\overrightarrow{1}, \hat{x}}\right)^{t} & 0
\end{array}\right]_{(3 \times 5)},
$$

while the bending part of the discrete strain-displacement operator related to the node ' $I$ ', $B_{b}^{l}$, takes the form

$$
B_{b}^{I}=\left[\begin{array}{cc}
N_{, \hat{x}}^{I}\left(t_{\hat{x}}\right)^{t} & N_{, \hat{x}}^{I}\left(x_{, \hat{x}}\right)^{t} \boldsymbol{\Lambda}_{I} \\
N_{, \hat{y}}^{I}\left(t_{\hat{y}}\right)^{t} & N_{, \hat{y}}^{I}\left(x_{, \hat{y}}\right)^{t} \boldsymbol{\Lambda}_{I} \\
N_{, \hat{x}}^{I}\left(t_{\hat{y}}\right)+N_{, \hat{y}}^{I}\left(t_{, \hat{x}}\right)^{t} & \left(N_{, \hat{x}}^{I}\left(x_{\hat{y}}\right)+N_{, \hat{y}}^{I}\left(x_{, \hat{x}}\right)^{t}\right) \boldsymbol{\Lambda}_{I}
\end{array}\right]_{(3 \times 5)} .
$$

According to the ANS approach, and using (27) and (46), the transverse shear part of (A4), in the local Cartesian frame, may be expressed as,

$$
B_{s}=\left[\begin{array}{llll}
B_{s \hat{x}}^{1} & B_{s \hat{x}}^{2} & B_{s \hat{x}}^{3} & B_{s \hat{x}}^{4} \\
B_{s \hat{y}}^{1} & B_{s \hat{y}}^{2} & B_{s \hat{y}}^{3} & B_{s \hat{y}}^{4}
\end{array}\right]_{(2 \times 20)},
$$

where the following transformation is implied

$$
\begin{aligned}
& B_{s \hat{x}}^{I}=\frac{\partial \xi}{\partial \hat{x}} B_{s \xi}^{I}+\frac{\partial \eta}{\partial \hat{x}} B_{s \eta}^{I}, \\
& B_{s \hat{y}}^{I}=\frac{\partial \xi}{\partial \hat{y}} B_{s \xi}^{I}+\frac{\partial \eta}{\partial \hat{y}} B_{s \eta}^{I} .
\end{aligned}
$$

The transformation parameters follows from (45). In (A8) the transverse shear part of the discrete strain-displacement operator is evaluated in the direction of the coordinate $\xi$

$$
\begin{aligned}
& B_{s \xi}^{1}=\frac{1}{4}(1-\eta)\left[-\left(t_{A}\right)^{t} \mid\left(x_{A, \xi}\right)^{t} \Lambda_{1}\right]_{(1 \times 5)}, \\
& B_{s \xi}^{2}=\frac{1}{4}(1-\eta)\left[\left(t_{A}\right)^{t} \mid\left(x_{A, \xi}\right)^{t} \Lambda_{2}\right]_{(1 \times 5)}, \\
& B_{s \xi}^{3}=\frac{1}{4}(1+\eta)\left[\left(t_{C}\right)^{t} \mid\left(x_{C, \xi}\right)^{t} \Lambda_{3}\right]_{(1 \times 5)}, \\
& B_{s \xi}^{4}=\frac{1}{4}(1+\eta)\left[-\left(t_{C}\right)^{t} \mid\left(x_{C, \xi}\right)^{t} \Lambda_{4}\right]_{(1 \times 5)},
\end{aligned}
$$

while in the direction of the coordinate $\eta$

$$
\begin{aligned}
& B_{s \eta}^{1}=\frac{1}{4}(1-\xi)\left[-\left(t_{D}\right)^{t} \mid\left(x_{D, \eta}\right)^{t} \Lambda_{1}\right]_{(1 \times 5)}, \\
& B_{s \eta}^{2}=\frac{1}{4}(1+\xi)\left[-\left(t_{B}\right)^{t} \mid\left(x_{B, \eta}\right)^{t} \Lambda_{2}\right]_{(1 \times 5)}, \\
& B_{s \eta}^{3}=\frac{1}{4}(1+\xi)\left[\left(t_{B}\right)^{t} \mid\left(x_{B, \eta}\right)^{t} \Lambda_{3}\right]_{(1 \times 5)}, \\
& B_{s \eta}^{4}=\frac{1}{4}(1-\xi)\left[\left(t_{D}\right)^{t} \mid\left(x_{D, \eta}\right)^{t} \Lambda_{4}\right]_{(1 \times 5)^{*}} .
\end{aligned}
$$

The $\xi$ and $\eta$ derivatives of the mid-surface position vector at the deformed configuration, $x_{, \xi}$ and $x_{i, \eta}$, which appear in expressions (A9) and (A10), are easily evaluated at the points A, B, C, D (Fig. 2)

$$
\begin{aligned}
& x_{A, \xi}=\frac{1}{2}\left(x_{2}-x_{1}\right), \\
& x_{C, \xi}=\frac{1}{2}\left(x_{3}-x_{4}\right), \\
& x_{B, \eta}=\frac{1}{2}\left(x_{3}-x_{2}\right), \\
& x_{D, \eta}=\frac{1}{2}\left(x_{4}-x_{1}\right),
\end{aligned}
$$

Similarly the position of the shell director at the deformed configuration $t$ may be obtained at the transverse shear points $A, B, C, D$ as

$t_{A}=\frac{1}{2}\left(t_{2}+t_{1}\right)$,

$t_{C}=\frac{1}{2}\left(t_{3}+t_{4}\right)$,

$t_{B}=\frac{1}{2}\left(t_{3}+t_{2}\right)$,

$t_{D}=\frac{1}{2}\left(t_{4}+t_{1}\right)$.

The transverse shear part of strain-displacement matrix, related to the local Cartesian frame, is thus evaluated through (A8-A12) and the evaluation of the variation of transverse strains at the points $\mathrm{A}, \mathrm{B}, \mathrm{C}, \mathrm{D}$ is avoided.

\section{A.3}

\section{Material stiffness matrix}

From (38) and (40) the material part of the stiffness matrix for isotropic material is recovered

${ }^{M} K^{I I}=\int_{S_{o}^{\varepsilon}}\left(B^{I}\right)^{t} H B^{I} \mathrm{~d} S_{o}$,

where $B^{\prime}$ follows from (A5-A7)

$B^{I}=\left[\begin{array}{c}B_{m}^{I} \\ B_{b}^{I} \\ B_{s}^{I}\end{array}\right]$

and $\mathrm{H}$ is given in $(\mathrm{A} 1-2)$. 


\section{A.4}

\section{Geometrical stiffness matrix}

From (38) and (41) the geometrical stiffness matrix may be conveniently expressed as a sum of membrane, bending and shear contributions,

$$
\begin{aligned}
{ }^{G} K^{I J}= & \int_{S_{c}^{\varepsilon}}\left\{\left[\begin{array}{cc}
{ }^{G} K_{m}^{I J} & 0_{(3 \times 2)} \\
0_{(2 \times 3)} & 0_{(2 \times 2)}
\end{array}\right]\right. \\
& \left.+\left[\begin{array}{ll}
0_{(3 \times 3)} & { }^{G} K_{b 1}^{I I} \\
{ }^{G} K_{b 2}^{I I} & { }^{G} K_{b 3}^{I I}
\end{array}\right]+\left[\begin{array}{ll}
0_{(3 \times 3)} & { }^{G} K_{s 1}^{I I} \\
{ }^{G} K_{s 2}^{I I} & { }^{G} K_{s 3}^{I I}
\end{array}\right]\right\} \mathrm{d} S_{\diamond},
\end{aligned}
$$

\section{A.4.1}

\section{Membrane part}

From (38) and (41) the sub-matrix related to the membrane contribution has a simple diagonal form

$$
{ }^{G} K_{m}^{I I}=\tilde{n}^{I I} I_{(3 \times 3)}
$$

where the following notation has been introduced

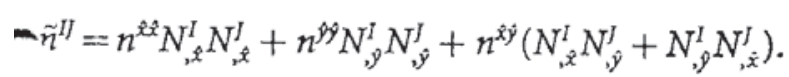

\section{A.4.2}

\section{Bending part}

The sub-matrices related to the bending contribution take the following forms

$$
\begin{aligned}
& { }^{G} K_{b 1}^{I I}=\tilde{m}^{I J} \Lambda_{y}, \\
& { }^{G} K_{b 2}^{I J}=\tilde{m}^{I I}\left(\Lambda_{1}\right)^{t}, \\
& { }^{G} K_{b 3}^{I J}=\left\{\begin{array}{cc}
\bar{m}^{\hat{x} I} X_{\dot{x}}^{I}+\bar{m}^{\hat{y}} Y_{\hat{y}}^{I} & \text { if } I=J, \\
0_{(2 \times 2)} & \text { if } I \neq J,
\end{array}\right.
\end{aligned}
$$

where

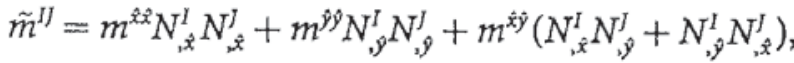

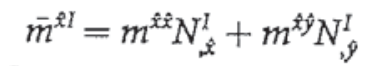

$$
\bar{m}^{\hat{y I}}=m^{\hat{y} \hat{y}} N_{, p}^{I}+m^{\chi \hat{y}} N_{, \hat{x}}^{I}
$$

In the third sub-matrix ${ }^{G} K_{b 3}^{I I}$ the second directional derivative of the shell director, $\mathrm{D} \delta t[\Delta t]$, is multiplied by base vectors at the deformed configuration (expressed in the Cartesian frame) defined by $x_{, x}$ and $x_{, y}$. The result may be expressed in the matrix form as

$$
r_{\dot{x}}^{I}=\left[\begin{array}{cc}
-x_{, \hat{x}}^{2} \sin \left(\omega^{I}\right) \cos \left(\psi^{I}\right) & -x_{, \hat{x}}^{2} \cos \left(\omega^{I}\right) \sin \left(\psi^{I}\right) \\
-\hat{x}_{, \hat{x}}^{3} \sin \left(\omega^{I}\right) \sin \left(\psi^{I}\right) & +x_{, \hat{x}}^{3} \cos \left(\omega^{I}\right) \cos \left(\psi^{I}\right) \\
\operatorname{sYMM} & -x_{, \hat{x}}^{1} \cos \left(\omega^{I}\right) \\
& -x_{, \hat{x}}^{2} \sin \left(\omega^{I}\right) \cos \left(\psi^{I}\right) \\
& -x_{, \hat{x}}^{3} \sin \left(\omega^{I}\right) \sin \left(\psi^{I}\right)
\end{array}\right]_{(2 \times 2)},
$$

$$
Y_{\hat{y}}^{I}=\left[\begin{array}{cc}
-x_{\hat{y}}^{2} \sin \left(\omega^{I}\right) \cos \left(\psi^{I}\right) & -x_{\hat{y}}^{2} \cos \left(\omega^{I}\right) \sin \left(\psi^{I}\right) \\
-\hat{x}_{\hat{y}}^{3} \sin \left(\omega^{I}\right) \sin \left(\psi^{I}\right) & +x_{, y}^{3} \cos \left(\omega^{I}\right) \cos \left(\psi^{I}\right) \\
\text { SYMM } & -x_{\hat{y}}^{1} \cos \left(\omega^{I}\right) \\
& -x_{\hat{y}}^{2} \sin \left(\omega^{I}\right) \cos \left(\psi^{I}\right) \\
& -x_{y, y}^{3} \sin \left(\omega^{I}\right) \sin \left(\psi^{I}\right)
\end{array}\right]_{(2 \times 2)},
$$

In (A21) and (A22) the notation

$$
x_{, \hat{t}}=\left(x_{, \hat{x}}^{1}, x_{, \hat{x}}^{2}, x_{, \hat{x}}^{3}\right)^{t}, \quad x_{, y}=\left(x_{, y}^{1}, x_{, \hat{y}}^{2}, x_{, y}^{3}\right)^{t} .
$$

is used.

\section{A.4.3}

\section{Transverse shear part}

The sub-matrices related to the transverse shear effects have the following forms

${ }^{G} K_{s 1}^{I I}=\left\{\begin{array}{cl}\bar{q}^{I I} \boldsymbol{\Lambda}_{I} & \text { if } I=J, \\ -\bar{q}^{I I} \boldsymbol{\Lambda}_{J} & \text { if } I \neq J .\end{array}\right.$

${ }^{G} K_{s 2}^{I J}=\bar{q}^{I I}\left(\Lambda_{I}\right)^{t}$,

The components $\bar{q}^{I J}$ are explicitly given as

$\bar{q}^{11}=\frac{1}{8}\left(-(1-\eta) q^{\xi}-(1-\xi) q^{\eta}\right)$,

$\bar{q}^{12}=\frac{1}{8}(1-\eta) q^{\xi}$,

$\bar{q}^{13}=0$,

$\bar{q}^{14}=\frac{1}{8}(1-\xi) q^{\eta}$,

$\bar{q}^{22}=\frac{1}{8}\left((1-\eta) q^{\xi}-(1+\xi) q^{\eta}\right)$,

$\bar{q}^{23}=\frac{1}{8}(1+\xi) q^{\eta}$,

$\vec{q}^{24}=0$,

$\bar{q}^{-33}=\frac{1}{8}\left((1+\eta) q^{\xi}+(1+\xi) q^{\eta}\right)$,

$\bar{q}^{34}=-\frac{1}{8}(1+\eta)$

$\bar{q}^{44}=\frac{1}{8}\left(-(1+\eta) q^{\xi}+(1-\xi) q^{\eta}\right)$

The diagonal sub-matrices ${ }^{G} K_{s 3}^{I I}$ take the following form

$$
{ }^{G} K_{s 3}^{11}=\left[\begin{array}{cc}
-\tilde{q}_{A D}^{2} \sin \left(\omega^{1}\right) \cos \left(\psi^{1}\right) & -\tilde{q}_{A D}^{2} \cos \left(\omega^{1}\right) \sin \left(\psi^{1}\right) \\
-\tilde{q}_{A D}^{3} \sin \left(\omega^{1}\right) \sin \left(\psi^{1}\right) & +\tilde{q}_{A D}^{3} \cos \left(\omega^{1}\right) \cos \left(\psi^{1}\right) \\
\operatorname{sYMM} & -\tilde{q}_{A D}^{1} \cos \left(\omega^{1}\right) \\
& -\tilde{q}_{A D}^{2} \sin \left(\omega^{1}\right) \cos \left(\psi^{1}\right) \\
& -\tilde{q}_{A D}^{3} \sin \left(\omega^{1}\right) \sin \left(\psi^{1}\right)
\end{array}\right]_{(2 \times 2)},
$$


${ }^{G} K_{s 3}^{22}=\left[\begin{array}{cc}-\tilde{q}_{A B}^{2} \sin \left(\omega^{2}\right) \cos \left(\psi^{2}\right) & -\tilde{q}_{A B}^{2} \cos \left(\omega^{2}\right) \sin \left(\psi^{2}\right) \\ -\tilde{q}_{A B}^{3} \sin \left(\omega^{2}\right) \sin \left(\psi^{2}\right) & +\tilde{q}_{A B}^{3} \cos \left(\omega^{2}\right) \cos \left(\psi^{2}\right) \\ & -\tilde{q}_{A B}^{1} \cos \left(\omega^{2}\right) \\ S Y M M & -\tilde{q}_{A B}^{2} \sin \left(\omega^{2}\right) \cos \left(\psi^{2}\right) \\ & -\tilde{q}_{A B}^{3} \sin \left(\omega^{2}\right) \sin \left(\psi^{2}\right)\end{array}\right]_{(2 \times 2)}$

(A28)

${ }^{G} K_{s 3}^{33}=\left[\begin{array}{cc}-\tilde{q}_{C B}^{2} \sin \left(\omega^{3}\right) \cos \left(\psi^{3}\right) & -\tilde{q}_{C B}^{2} \cos \left(\omega^{3}\right) \sin \left(\psi^{3}\right) \\ -\tilde{q}_{C B}^{3} \sin \left(\omega^{3}\right) \sin \left(\psi^{3}\right) & +\tilde{q}_{C B}^{3} \cos \left(\omega^{3}\right) \cos \left(\psi^{3}\right) \\ & -\tilde{q}_{C B}^{1} \cos \left(\omega^{3}\right) \\ S Y M M & -\tilde{q}_{C B}^{2} \sin \left(\omega^{3}\right) \cos \left(\psi^{3}\right) \\ & -\tilde{q}_{C B}^{3} \sin \left(\omega^{3}\right) \sin \left(\psi^{3}\right)\end{array}\right]_{(2 \times 2)}$,

${ }^{G} K_{s 3}^{44}=\left[\begin{array}{cc}-\tilde{q}_{C D}^{2} \sin \left(\omega^{4}\right) \cos \left(\psi^{4}\right) & -\tilde{q}_{C D}^{2} \cos \left(\omega^{4}\right) \sin \left(\psi^{4}\right) \\ -\tilde{q}_{C D}^{3} \sin \left(\omega^{4}\right) \sin \left(\psi^{4}\right) & +\tilde{q}_{C D}^{3} \cos \left(\omega^{4}\right) \cos \left(\psi^{4}\right) \\ & -\tilde{q}_{C D}^{1} \cos \left(\omega^{4}\right) \\ S Y M M & -\tilde{q}_{C D}^{2} \sin \left(\omega^{4}\right) \cos \left(\psi^{4}\right) \\ & -\tilde{q}_{A D}^{3} \sin \left(\omega^{4}\right) \sin \left(\psi^{4}\right)\end{array}\right]_{(2 \times 2)}$

and

${ }^{G} K_{s 3}^{I I}=0 \quad$ if $\quad I \neq J$.

The vectors $\tilde{q}_{A B}, \tilde{q}_{C B}, \tilde{q}_{C D}$ and $\tilde{q}_{A D}$, in $(A 27-30)$ are defined as

$\tilde{q}_{A D}=\frac{1}{4}\left((1-\eta) x_{A, \xi} q^{\xi}+(1-\xi) x_{D, \eta} q^{\eta}\right)$,

$\tilde{q}_{A B}=\frac{1}{4}\left((1-\eta) x_{A, \xi} q^{\xi}+(1+\xi) x_{B, \eta} q^{\eta}\right)$,

$\tilde{q}_{C B}=\frac{1}{4}\left((1+\eta) x_{C, \xi} q^{\zeta}+(1+\xi) x_{B, \eta} q^{\eta}\right)$,

$\tilde{q}_{C D}=\frac{1}{4}\left((1+\eta) x_{C, \xi} q^{\xi}+(1-\xi) x_{D, \eta} q^{\eta}\right)$.

Remark A.1 Note, that the stress resultants in curvilinear coordinate system $\left(q^{\xi}\right.$ and $\left.q^{\eta}\right)$ take part in the transverse shear part of the geometric stiffness matrix (A24-32) and not the stress resultants in the local Cartesian system, $q^{\hat{x}}$ and $q^{\hat{\psi}}$, as one would expect. Therefore, the following transformation

$q^{\xi}=\frac{\partial \xi}{\partial \hat{x}} q^{\hat{x}}+\frac{\partial \xi}{\partial \hat{y}} q^{\hat{y}}, \quad q^{\eta}=\frac{\partial \eta}{\partial \hat{x}} q^{\hat{x}}+\frac{\partial \eta}{\partial \hat{y}} q^{\vartheta}$,

is required.

\section{Appendix B}

B.1

\section{Constitutive equatiojns for multilayered shell}

Fibre-reinforced composites are composed of oriented fibers embedded in a matrix. The basic characteristic of composite with oriented fibers in its anisotropic behaviour. When shell consists of several laminae with varying (anisotropic) material properties it is called a laminated composite shell. It is common practice to represent mechanical behaviour of the laminated shell on the macroscopic level as a layer-wise homogeneous and anisotropic continuum. At small strains the behavior of a composite at a particular layer can be assumed as purely elastic and described with a simple hyperelastic orthotropic material law. By assuming that layers are perfectly bonded together, the matrix $\mathrm{H},(\mathrm{A} 3)$, takes a form

$\mathrm{H}=\left[\begin{array}{ccc}\overline{\mathrm{H}}_{m} & \overline{\mathrm{H}}_{m b} & 0 \\ & \overline{\mathrm{H}}_{b} & 0 \\ \mathrm{SYMM} & & \overline{\mathrm{H}}_{s}\end{array}\right]_{(8 \times 8)}$.

The submatrices may be obtained from

$\overline{\mathrm{H}}_{m}=\sum_{K=1}^{N_{\text {layt }}} \int_{h_{K}^{-}}^{h_{K}^{+}} \mathrm{C}_{K} \mathrm{~d} \xi, \quad \overline{\mathrm{H}}_{m b}=\sum_{K=1}^{N_{\text {lagr }} h_{K}^{+}} \int_{h_{K}^{-}}^{+} \mathrm{C}_{K} \xi \mathrm{d} \xi$,
$\overline{\mathrm{H}}_{b}=\sum_{K=1}^{N_{\text {lary }}} \int_{h_{K}^{-}}^{h_{K}^{+}} \mathrm{C}_{K} \xi^{2} \mathrm{~d} \xi, \quad \overline{\mathrm{H}}_{s}=\sum_{K=1}^{N_{\text {lagr }}} \int_{h_{K}^{+}}^{+} \mathrm{C}_{s K} \mathrm{~d} \xi$.

In (B2) $N_{\text {layr }}$ is a number of layers of the composite shell, while $h_{K}^{-}$and $h_{K}^{+}$(with $h_{K}^{+}>h_{K}^{-}$) are values of $\xi$ parameter as the edges of $K$-th layer. Clearly $\left|h_{K}^{+}-h_{K}^{-}\right|$is the thickness of $K$-th layer.

With the assumption that throught the thickness metrics is constant, i.e. $G_{\alpha \beta} \approx A_{\alpha \beta}$, the integrals in (B2) can be easily evaluated. In that case the matrices $C_{K}$ and $C_{s K}$ (which consist of the components of the fourth order symmetric tensor $\mathrm{C}_{K}^{i j k l}$, $i j \neq 33, k l \neq 33$ )

$C_{K}=\left[\begin{array}{ccc}C_{K}^{1111} & C_{K}^{1122} & C_{K}^{1112} \\ & C_{K}^{2222} & C_{K}^{2212} \\ S Y M M & & C_{K}^{1212}\end{array}\right]_{(3 \times 3)}, C_{s K}=\left[\begin{array}{cc}C_{K}^{1313} & C_{K}^{1323} \\ S Y M M & C_{K}^{2323}\end{array}\right]_{(2 \times 2)}$,

are considered to be constant throughout the layer $K$.

To define the components of $C_{K}$ and $C_{s K}$ a local Cartesian frame with axes $\left(\hat{x}_{K}^{*}, \hat{y}_{K}^{*}, \hat{z}_{K}^{*}\right)$ (which coincide with the axes of material orthotropy) and unit base vectors $\left(\hat{e}_{1}^{*}, \hat{e}_{2}^{*}, \hat{e}_{3}^{*}\right)$, must be specified at layer $K$ at the integration point. Since $\hat{e}_{3}^{*}$ and $\hat{e}_{3},(48)$, coincide, the transformation between two local Cartesian frames is uniquely defined by an angle $\theta_{K}$, which is measured counterclockwise from $\hat{e}_{1}^{*}$ to $\hat{e}_{1}$.

The components of the fourth order tensor transform as $C_{K}^{i j k i}=T_{i m} T_{j n} C_{K}^{* \text { mnop }} T_{k o} T_{l p}$, where $T_{m i}=\hat{e}_{m} \cdot \hat{e}_{i}^{*}$. The following relations are obtained (details are provided in any textbook on the mechanics of composite laminates, e.g. Matthews and Rawlings (1994), Chapter 7)

$$
\begin{aligned}
& C_{K}^{1111}=c^{4} C_{K}^{* 1111}+2 c^{2} s^{2}\left(C_{K}^{* 1122}+2 C_{K}^{* 1212}\right)+s^{4} C_{K}^{* 2222}, \\
& C_{K}^{1112}=c^{2} s^{2}\left(C_{K}^{* 1111}+C_{K}^{* 2222}-4 C_{K}^{* 1212}\right)+\left(c^{4}+s^{4}\right) C_{K}^{* 1122}, \\
& C_{K}^{1112}=c s\left[c^{2} C_{K}^{* 1111}-s^{2} C_{K}^{* 2222}-\left(c^{2}-s^{2}\right)\left(C_{K}^{* 1122}+2 C_{K}^{* 1212}\right)\right] \\
& C_{K}^{2222}=s^{4} C_{K}^{* 1111}+2 c^{2} s^{2}\left(C_{K}^{* 1122}+2 C_{K}^{* 1212}\right)+c^{4} C_{K}^{* 2222}, \\
& C_{K}^{2212}=c s\left[s^{2} C_{K}^{* 1111}-c^{2} C_{K}^{* 2222}+\left(c^{2}-s^{2}\right)\left(C_{K}^{* 1122}+2 C_{K}^{* 1212}\right)\right],
\end{aligned}
$$




$$
\begin{aligned}
& C_{K}^{1313}=c^{2} C_{K}^{* 1313}+s^{2} C_{K}^{* 2323}, \\
& C_{K}^{1323}=c s\left(C_{K}^{* 1313}-C_{K}^{* 2323}\right), \\
& C_{K}^{2323}=c^{2} C^{* 2323}+s^{2} C^{* 1313},
\end{aligned}
$$$$
C_{K}^{1212}=c^{2} s^{2}\left(C_{K}^{* 1111}+C_{K}^{* 2222}-2 C_{K}^{* 1122}\right)+\left(c^{2}-s^{2}\right)^{2} C_{K}^{* 1212},
$$

with $c=\cos \left(\theta_{K}\right)$ and $s=\sin \left(\theta_{K}\right)$. The components of the material tensor along the material axes of orthotropy are obtained by a condensation of the three dimensional orthotropic (Green) model for small strains

$$
\mathrm{C}_{K}^{* 1111}=\frac{E_{1}}{1-v_{12}^{2} \frac{E_{2}}{E_{1}}}, \quad C_{K}^{* 1122}=\frac{v_{12} E_{2}}{1-v_{12}^{2} \frac{E_{2}}{E_{1}}}, \quad C_{K}^{* 2222}=\frac{E_{2}}{1-v_{12}^{2} \frac{E_{2}}{E_{1}}},
$$

$$
C_{K}^{* 1212}=G_{12}, \quad C_{K}^{* 1313}=\kappa_{1} G_{13}, \quad C_{K}^{* 2323}=\kappa_{2} G_{23} \text {. }
$$

where $\kappa_{1}$ and $\kappa_{2}$ are the shear correction factors for laminated composite shell.

Eemark B.I We emphasise that Appendix B contains all required changes that make the present computational shell model suitable for simulation of multilayered composite shells. In this case, matrix from expressions (A3) and (A13) can still be used, with the matrix $\mathrm{H}$ defined as in (BI).

\section{References}

Andelfinger, U.; Ramm, E. 1993: EAS-elements for two-dimensional, three-dimensional, plate and shell structures and their equivalence to HR-elements. Int. J. Numer. Meth. Engng. 36: 1311-1337

Bașar, Y.; Ding, Y. 1990: Theory and finite-element formulation for shell structures undergoing finite rotations, In: Voyiadjis, G. Z.; Karamanlidis, D. (eds.): Advances in the Theory of Plates and Shells. Elsevier Science Publ.

Bathe, K.-J.; Dvorkin, E. N. 1985: A four-node plate bending element based on Mindlin/Reissner plate theory and a mixed formulation. Int. J. Numer. Meth. Engng. 21: 367-383

Brank, B.; Perić, D.; Damjanić, F. B. 1993: A nonlinear four node shell element with explicit integration and elementary rotations. In: Kussmaul, K. F. (ed.): Proc. Trans. of the 12th Int. Conf. SMIRT, Vol. B, 153 - 158, Elsevier Science Publ.

iichter, N.; Ramm, E. 1992: Shell theory versus degeneration-a mparison in large rotation finite element analysis. Int. J. Numer. Meth. Engng. 34: 39-59

Crisfield, M. A. 1991: Non-linear Finite Element Analysis of Solids and Structures, Vol. 1. Chichester: John Wiley

Dvorkin, E. N.; Bathe, K.-J. 1984: A continuum mechanics based four-node shell element for general non-linear analysis. Eng. Comput. 1: $77-88$

Figueiras, J. A.; Owen, D. R. J. 1984: Analysis of elasto-plastic and geometrically nonlinear anisotropic plates and shells. In: Hinton, E.;
Owen, D. R. J. (eds.): Finite Element Software for Plates and Shells, 235-326, Swansea: Pineridge Press

Gebhardt, H.; Schweizerhof, K. 1993: Interpolation of curved shell geometries by low order finite elements-errors and modifications. Int. J. Num. Meth. Engng. 36: 287-302

Green, A. E.; Zerna, W. 1968: Theoretical Elasticity. 2nd Ed. Oxford University Press

Laschet, G.; Jeusette, J.-P. 1990: Postbuckling finite element analysis of composite panels. Compos. Struct. 14: $35 \sim 48$

Lindberg, G. M.; Olson, M. D.; Cowper, G. R. 1969: New developments in the finite element analysis of shells. National Research Council of Canada, Quarterly Bulletin of the Division of Mechanical Engineering and National Aeronautical Establishment, 4: 1-38

Matthews, F. L.; Rawlings, R. D. 1994: Composite Materials:

Engineering and Science. London: Chapman and Hall

Naghdi, P. M. 1972: The theory of shells. In: Flüge, S. (ed.): Handbuch der Physik, Vol. VI/2, SPringer-Verlag

Parisch, H. 1991: An investigation of a finite rotation four node assumed strain element. Int. J. Num. Meth. Engng. 31: 127-150

Perić, D.; Owen, D. R. J. 1991: The Morley thin shell finite element for large deformations problems: simplicity versus sophistication. In:

Bićanić, N. (ed.): Proc. Int. Conf. on Nonlinear Engineering Computations, 121-142, Swansea: Pineridge Press

Pian, T. H. H.; Sumihara, K. 1984: Rational approach for assumed stress finite elements. Int. J. Num. Meth. Engng. 20: 1685-1695

Ramm, E.; Matzenmiller, A. 1986: Large deformation shell analysis based on the degeneration concept. In: Hughes, T. J. R.; Hinton, E. (eds.): Finite Element Methods for Plate and Shell Structures, 365-393, Swansea: Pineridge Press

Saigal, S.; Kapania, R.; Yang, Y. 1986: Geometrically nonlinear finite element analysis of imperfect laminated shells. J. Composite Mater, 20: $197-214$

Sansour, C.; Bufler, H. 1992: An exact finite rotation shell theory, its mixed variational formulation and its finite element implementation. Int. ). Num. Meth. Engng. 34: 73-115

Simo, J. C.; Fox, D. D. 1989a: On a stress resultant geometrically exact shell model. Part I: Formulation and optimal parametrization. Comp. Meth. Appl. Mech. Engng. 72: 267-304

Simo, J. C.; Fox, D. D.; Rifai, M. S. 1989b: On a stress resultant geometrically exact shell model. Part II: The linear theory; Computational aspects. Comp. Meth. Appl. Mech. Engng. 73: 53-92 Simo, J. C.; Fox, D. D.; Rifai, M. S. 1990: On a stress resultant geometrically exact shell model. Part III: Computational aspects of the norlinear theory. Comp. Meth. Appl. Mech. Engng. 79: 21-70 Simo, J. C.; Hughes, T. J. R. 1986: On the variational foundations of assumed strain methods. J. Appl. Mech. 53: 51-54

Stander, N.; Matzenmiller, A.; Ramm, E. 1989: An assessment of assumed strain methods in finite rotation shell analysis. Eng. Comput. 6: $57-66$

Stanley, G. 1985: Continuum Based Shell Elements. Ph.D. Dissertation, Applied Mechanics Division, Stanford: Stanford University Timoshenko, S.; Woinowsky-Krieger, S. 1959: Theory of Plates and Shells, 2nd edition, New York: McGraw-Hill

Wriggers, P.; Gruttmann, F. 1993: Thin shells with finite rotations formulated in Biot stresses: Theory and finite element formulation. Int. J. Numer. Meth. Engng. 36: 2049-2071

Wriggers, P.; Simo, J. C. 1990: A general procedure for the direct computation of turning and bifurcation points. Int. J. Numer, Meth. Engng. 30: 155-176 